\title{
Gravitationally bound axions and how one can discover them
}

\author{
Xunyu Liang ${ }^{*}$ and Ariel Zhitnitsky ${ }^{\dagger}$ \\ Department of Physics \& Astronomy, University of British Columbia, Vancouver BC V6T1Z1, Canada
}

(Received 23 October 2018; published 24 January 2019)

\begin{abstract}
As recently advocated in [H. Fischer et al., Phys. Rev. D 98, 043013 (2018)], there is a fundamentally new mechanism for the axion production in the Sun and Earth. However, the role of very slow axions in previous studies were neglected because of its negligible contribution to the total axion production by this new mechanism. In the present work we specifically focus on analysis of the nonrelativistic axions which will be trapped by the Sun and Earth due to the gravitational forces. The corresponding emission rate of these low energy axions (below the escape velocity) is very tiny. However, these axions will be accumulated by the Sun and Earth during their life-times, i.e., 4.5 billion of years, which greatly enhances the discovery potential. The computations are based on the so-called axion quark nugget (AQN) dark matter (DM) model. This model was originally invented as a natural explanation of the observed ratio $\Omega_{\text {dark }} \sim \Omega_{\text {visible }}$ when the DM and visible matter densities assume the same order of magnitude values, irrespectively to the axion mass $m_{a}$ or initial misalignment angle $\theta_{0}$. This model, without adjustment of any parameters, gives a very reasonable intensity of the extreme UV (EUV) radiation from the solar corona as a result of the AQN annihilation events with the solar material. This extra energy released in corona represents a resolution, within AQN framework, a long standing puzzle known in the literature as the "solar corona heating mystery". The same annihilation events also produce the axions. The flux of these axions is unambiguously fixed in this model and expressed in terms of the EUV luminosity from solar corona. We make few comments on the potential discovery of these gravitationally bound axions.
\end{abstract}

DOI: $10.1103 /$ PhysRevD.99.023015

\section{INTRODUCTION}

The Peccei-Quinn (PQ) mechanism accompanied by the axions remains the most compelling resolution of the strong $C P$ problem, see original papers [1-3] and recent reviews [4-12] on the subject. We refer to the review articles for the discussions and analysis on the recent activities in the field of the axion searches by a numerous number of different groups using very different instruments.

For the purposes of the present work it is sufficient to mention that the conventional dark matter galactic axions are produced due to the misalignment mechanism [13] when the cosmological field $\theta(t)$ oscillates and emits cold axions before it settles down at its final destination $\theta_{\text {final }}=0$. Another mechanism is due to the decay of the topological objects [14-19]. There is a number of uncertainties and remaining discrepancies in the corresponding

\footnotetext{
xunyul@phas.ubc.ca

arz@physics.ubc.ca
}

Published by the American Physical Society under the terms of the Creative Commons Attribution 4.0 International license. Further distribution of this work must maintain attribution to the author(s) and the published article's title, journal citation, and DOI. Funded by SCOAP. estimates. We shall not comment on these subtleties ${ }^{1}$ by referring to the original papers [14-19]. It is important that in both cases the produced axions are nonrelativistic particles with typical $v_{\text {axion }} / c \sim 10^{-3}$, and their contribution to the dark matter density scales as $\Omega_{\text {axion }} \sim m_{a}^{-7 / 6}$. This scaling unambiguously implies that the axion mass must be fine-tuned $m_{a} \simeq 10^{-5} \mathrm{eV}$ to saturate the $\mathrm{DM}$ density today, see footnote 1 , while larger axion mass will contribute very little to $\Omega_{\mathrm{DM}}$. The cavity type experiments have a potential to discover these nonrelativistic axions.

Axions can be also produced as a result of the Primakoff effect in a stellar plasma at high temperature [20]. These axions are ultrarelativistic as the typical average energy of the axions emitted by the Sun is $\langle E\rangle=4.2 \mathrm{keV}$, see [21]. The searches for the solar axions are based on the helioscope instrument CAST (CERN Axion Search Telescope) [21].

\footnotetext{
${ }^{1}$ According to the most recent computations presented in Ref. [19], the axion contribution to $\Omega_{\mathrm{DM}}$ as a result of decay of the topological objects can saturate the observed DM density today if the axion mass is in the range $m_{a}=$ $(2.62 \pm 0.34) \times 10^{-5} \mathrm{eV}$, while the earlier estimates suggest that the saturation occurs at a larger axion mass. One should also emphasize that the computations [14-19] have been performed with the assumption that PQ symmetry was broken after inflation.
} 
It has been suggested in recent work [22] that there is a fundamentally novel mechanism of the axion production in the Sun. This mechanism is deeply rooted to the so-called axion quark nugget (AQN) dark matter model when the stability of the nuggets is supported by the axion domain wall. The most important consequence of the new production mechanism is that the emitted axions (from the axion domain wall when the nugget gets annihilated) will be released with relativistic velocities with typical values $v_{\text {axion }}^{\mathrm{AQN}} \simeq 0.5 c$. These features should be contrasted with conventional galactic nonrelativistic axions $v_{\text {axion }} \sim 10^{-3} c$ and solar ultrarelativistic axions with typical energies $\langle E\rangle=4.2 \mathrm{keV}$.

The computations in Ref. [22] of the spectral properties of the axions produced by this novel mechanism were based on the approximation which is known to be badly violated for low-energy part of the spectrum with $v \ll c$. This part of the spectrum represents very tiny portion of the produced axions. Therefore, it had been ignored in the original studies [22]. However, the upgraded CAST instrument will be highly sensitive to the low energy part of the spectrum. Therefore, it is highly desirable to develop a new computational technique which would allow us to carry out the computations in the region of small velocities $v \ll c$.

Furthermore, the low-energy axions produced in the Sun might be trapped by strong gravitational force such that $v \leq v_{\text {trapped }}$ will be trapped by the Sun indefinitely. The $v_{\text {trapped }}$ is numerically the same as the free fall velocity,

$$
\frac{v_{\text {trapped }}}{c}=\sqrt{\frac{2 G M_{\odot}}{c^{2} R_{\odot}}} \simeq 2 \times 10^{-3} .
$$

While the portion of these low energy axions is tiny as we shall estimate below, these trapped axions may play an important role in physics as they will be accumulated around the Sun during entire life time of the solar system, i.e., around 4.5 billion years. The effects related to the trapped axions are not new, and discussed previously in the literature [23]. The goal here is to present some numerical estimates for our specific AQN model when the axions which are produced as a result of the annihilation events in the solar atmosphere and will be indefinitely bounded to the Sun.

Therefore, the main goal of the present studies is to develop a new technique to generalize the results of Ref. [22] to perform the self-consistent computations of the axion spectrum in the regime when the axion velocities are small $v \ll c$. The corresponding generalization of the results [22] requires to abandon the so-called "thin wall approximation" and develop some new technical tools which proper describe the regime with $v \ll c$.

We should emphasize that the present work is a natural continuation of the previous studies [22]. Therefore, to avoid repetition we refer the readers to that paper with detail discussions of the AQN framework itself, its motivation, its consequences and predictions. The only comment we would like to make here is as follows. The AQN framework is consistent with all known astrophysical, cosmological, satellite and ground based constraints. In fact, in a number of cases some observables become very close to present day constraints. Furthermore, in a few cases the predictions of the model may explain a number of the long standing mysteries as overviewed in [22].

The paper is organized as follows. In Sec. II we develop a new technique which allows to generalize these computations for low energy portion of the spectrum when $v \ll c$. We use the corresponding results in Sec. III to discuss the physics of the trapped axions and we highlight the basic ideas how to discover them. We conclude in Sec. IV with a few thoughts on the future developments.

\section{AXIONS FROM AQNS: INTENSITY AND THE SPECTRUM}

The AQN model was invented long ago [24] (though a specific formation mechanism of the nuggets was developed in much more recent papers [25-27]) as a natural explanation of the observed ratio $\Omega_{\text {dark }} \sim \Omega_{\text {visible. In the }}$ context of the present work the argument supporting the AQN model goes as follows. It has been known for quite some time that the total intensity of the observed EUV and soft x-ray radiation (averaged over time) can be estimated as follows,

$$
L_{\odot \text { (from Corona) }} \sim 10^{30} \cdot \frac{\mathrm{GeV}}{\mathrm{s}} \sim 10^{27} \cdot \frac{\mathrm{erg}}{\mathrm{s}},
$$

which represents (since 1939) the renowned "the solar corona heating puzzle." The observations (2) imply that the corona has the temperature $T \simeq 10^{6} \mathrm{~K}$ which is 100 times hotter than the surface temperature of the Sun, and conventional astrophysical sources fail to explain the EUV and soft $\mathrm{x}$ ray radiation from corona.

It turns out that if one estimates the extra energy being produced within the AQN dark matter scenario one obtains the total extra energy $\sim 10^{27} \mathrm{erg} / \mathrm{s}$ which precisely reproduces (2) for the observed EUV and soft x-ray intensities [28]. The full scale MonteCarlo simulations [29] support this estimate. One should add that the estimate $\sim 10^{27} \mathrm{erg} / \mathrm{s}$ for extra energy is derived exclusively in terms of known dark matter density $\rho_{\mathrm{DM}} \sim 0.3 \mathrm{GeV} \mathrm{cm}^{-3}$ and dark matter velocity $v_{\mathrm{DM}} \sim 10^{-3} c$ surrounding the sun without adjusting any parameters of the model. We interpret this "numerical coincidence" as an additional hint supporting the AQN model. Our original remark relevant for the present work is that if one accepts the explanation that the solar corona heating puzzle is resolved within AQN scenario than the axion flux will be unambiguously fixed in terms of the EUV observed luminosity (2) as the axion field represents the crucial element in the AQN construction. 
We start our presentation with subsection II A where we highlight the basic results from Ref. [22] by providing a self-contained text for the convenience of the readers. In next subsections II B and II C we explain the computational framework and present the results of the computations, referring to Appendix A for the technical details.

\section{A. Intensity}

The axions play a key role in construction of the AQNs as they provide an additional pressure to stabilize the nuggets. The corresponding axion contribution into the total nugget's energy density has been computed in [27]. Depending on parameters the axion's contribution to the nugget's mass represents about $1 / 3$ of the total mass. It can be translated in terms of the axion luminosity from the Sun as follows [22]:

$$
L_{\odot(\text { axion })} \simeq 1.6 \times 10^{27} \cdot \frac{\mathrm{erg}}{\mathrm{s}} .
$$

The corresponding axion flux measured on Earth can be computed as follows [22]

$$
\begin{gathered}
\Phi(\text { solar axions }) \sim \frac{L_{\odot(\text { axion })}}{4 \pi\left\langle E_{a}\right\rangle D_{\odot}^{2}} \sim 0.3 \times 10^{17} \frac{1}{\mathrm{~cm}^{2} \mathrm{~s}}\left(\frac{10^{-5} \mathrm{eV}}{m_{a}}\right), \\
D_{\odot} \simeq 150 \times 10^{6} \mathrm{~km},
\end{gathered}
$$

where we assume that the axion's energy when the antinuggets get annihilated is slightly relativistic $E_{a} \simeq 1.2 m_{a}$, but never becomes very relativistic. The corresponding energy flux is [22]

$$
m_{a} \Phi(\text { solar axions }) \sim 3 \times 10^{11} \frac{\mathrm{eV}}{\mathrm{cm}^{2} \mathrm{~s}} .
$$

These estimates should be compared with conventional cold dark matter galactic axion contribution assuming the axions saturate the observed DM density:

$$
\begin{aligned}
m_{a} \Phi(\text { galactic axions }) \sim \rho_{\mathrm{DM}} \cdot v_{\mathrm{DM}} & \simeq \frac{0.3 \mathrm{GeV}}{\mathrm{cm}^{3}} v_{\mathrm{DM}} \\
& \simeq 10^{16} \frac{\mathrm{eV}}{\mathrm{cm}^{2} \mathrm{~s}} .
\end{aligned}
$$

Similar estimates can be also carried out for Earth. In this case as explained in [22] the observations of the Electricity and Magnetism $(E \& M)$ showers due to the nuggets entering the Earth's atmosphere (before hitting the Earth's surface) require very large area detectors. The nuggets will continue to radiate $E \& M$ energy in the deep underground. However, this radiation by obvious reasons cannot be recovered and observed. At the same time the observation of the axions (which have been produced as a result of the annihilation events in the very deep underground) is possible, and in fact very promising.
Indeed, the corresponding axion flux can be estimated as follows [22]

$$
m_{a} \Phi(\text { Earth axions }) \sim 10^{16}\left(\frac{\Delta B}{B}\right) \frac{\mathrm{eV}}{\mathrm{cm}^{2} \mathrm{~s}},
$$

where $\Delta B / B$ is the portion of the AQNs which get annihilated in the Earth's interior. Interestingly, the axion flux (7) which is generated due to the AQN annihilation events is much larger than the flux (5) generated due to the AQN annihilation events in the solar corona and measured on Earth. At the same time, the axion flux (7) is the same order of magnitude as the conventional cold dark matter galactic axion contribution (6). This is because the parameter $\Delta B / B \sim 1$ is expected to be order of one, as a finite portion of the AQNs will get annihilated in the Earth's interior, which includes all components: the crust, the mantle and the core. However, the wave lengths of the axions produced due to $\mathrm{AQN}$ annihilations, are much shorter due to their relativistic velocities $v \sim 0.5 c$, in contrast with conventional galactic isotropic axions with $v \sim 10^{-3} c$. Therefore, these two distinct contributions can be easily discriminated.

\section{B. Spectral properties. General comments}

The basic idea of the computation of the spectrum is as follows. Consider an AQN losing its mass due to the annihilation with surrounding material, while that the axion portion to the energy remains the same, as it is not linked to the annihilation processes. One should comment here that the axion domain wall in the equilibrium does not emit any axions as a result of pure kinematical constraint: the domain wall axions are off-shell axions in the equilibrium. The time dependent perturbation obviously changes this equilibrium configuration. In other words, the configuration becomes unstable because the total energy of the system is no longer at its minimum. To retrieve its ground state, an AQN will therefore intend to lower its domain wall mass by radiating the axions. To summarize: the emission of axions is an inevitable consequence during the annihilation of antinuggets in simply for the reason to maintain the AQN stability.

Now, we want to identify a precise mechanism which produces the on-shell freely propagating axions emitted by the axion domain wall. In this section we overview the basic idea of the computational technique to be used. To address this question, we consider the general form of a domain wall solution:

$$
\phi\left(R_{0}\right)=\phi_{w}\left(R_{0}\right)+\chi
$$

where $R_{0}$ is the radius of the $\mathrm{AQN}, \phi_{w}$ is the classical solution of the domain wall, while $\chi$ describes the excitations due to the time dependent perturbation. We should note that, $\phi_{w}$ is clearly off-shell classical solution, while $\chi$ 
describes the on-shell propagating axions. Thus, whenever the domain wall is excited, namely $\chi \neq 0$, freely propagating axions will be produced and emitted by the excitation modes.

Few comments are in order before we proceed in subsection IIC with description of the technical details and corresponding results. First, if the domain wall can be considered to be infinitely large in $x y$ direction such that the profile function depends on a single variable $z$ the computations can be carried out easily as the classical profile function $\phi_{w}(z)$ is known exactly. This is precisely the procedure which has been adopted in previous paper [22]. The corresponding technique is justified when a typical size $L_{x} \sim L_{y} \gg m_{a}^{-1}$ along $x, y$ is much larger than the width of the domain wall of order $m_{a}^{-1}$. If the wave length of the emitted axion is small, i.e., $\lambda_{a} \sim m_{a}^{-1}$ the axions cannot carry any information about the finite size of the system and the approximation is marginally justified $\left(\lambda_{a}\right.$ stands for the de Brogile wavelength of the emitted axion). This is precisely the approximation, the so-called "thin wall approximation" which has been adopted in computations [22]. This approximate treatment is marginally justified for relativistic axions with $v \sim c$, and we expect that accounting for the finite size of the system cannot drastically change the results in the relativistic domain $v \sim c$. This will be explicitly confirmed below by present computations accounting for finite size of the system.

Second, it is quiet obvious that the "thin wall approximation" is badly broken for nonrelativistic axions with $v \ll c$ when $\lambda_{a} \gg m_{a}^{-1}$ and a new technique must be developed for proper analysis. The basic idea of computation accounting for finite size of the system $R$ goes as follows. Suppose an AQN is traveling in vacuum where no annihilation event takes place, we expect the solution stays in its ground state $\phi\left(R_{0}\right)=\phi_{w}\left(R_{0}\right)$ which corresponds to the minimum energy state. Since there is no excitation (i.e., $\chi=0$ ), no free axion can be produced. However, the scenario drastically changes when some baryon charge from the AQN get annihilated. Due to these annihilation processes, the AQN starts to loose a small amount of its mass, and consequentially its size shrinks from $R_{0}$ to a slightly smaller radius $R_{\text {new }}=R_{0}-\Delta R$. Note that its quantum state $\phi\left(R_{0}\right)=\phi_{w}\left(R_{0}\right)$ is no longer the ground state, because a lower energy state $\phi_{w}\left(R_{\text {new }}\right)$ becomes available. Then, we may write the current state of the domain wall as $\phi\left(R_{0}\right)=\phi_{w}\left(R_{\text {new }}\right)+\phi_{w}^{\prime}\left(R_{\text {new }}\right) \Delta R$, so the domain wall now has a nonzero exciting mode $\chi=$ $\phi_{w}^{\prime}\left(R_{\text {new }}\right) \Delta R$ and free axions can be produced during oscillations of the domain wall. To reiterate: the annihilation of antinuggets with surrounding matter forces the domain wall to oscillate. These domain wall oscillations generate excitation modes which ultimately lead to radiation of the propagating axions.

Our last comment deals with terminology and notations. The results for the spectrum obtained using the thin-wall approximation is coined as the 1D spectrum. As we mentioned above it is marginally justified when $\lambda_{a} \sim m_{a}^{-1}$, and it admits mathematically exact treatment which was previously presented in [22]. In the present work we mostly deal with $3 \mathrm{D}$ computations when a finite size of the system plays a key role, which is always the case for $\lambda_{a} \gg m_{a}^{-1}$. The potential pitfall is that some technical simplifications are required to treat the $3 \mathrm{D}$ case. Consequentially, the obtained results might be sensitive to these technical simplifications. In order to characterize the sensitivity to our technical simplifications we introduce a tunable parameter $\delta$ which varies from 0 to 1 , so $\delta$ will serve as a probe to test the sensitivity with respect to numerical simplifications. As we shall see below, the obtained results are not very sensitive to the choice of $\delta$. Therefore we conclude that our 3D results are robust and reliable.

In what follows we will express the normalized spectrum as a function of the speed of emitted axion $v_{a} / c$ defined in the nugget's frame, defined as follows

$\rho\left(v_{a}\right) \equiv \frac{1}{\Phi_{\text {axions }}^{\text {tot }}} \frac{d}{d v_{a}} \Phi_{\text {axions }}\left(v_{a}\right), \quad \int_{0}^{1} d v_{a} \rho\left(v_{a}\right)=1$,

where $\Phi_{\text {axions }}^{\text {tot }}$ is the axion flux inserted to Eq. (9) for normalization purposes. It assumes the magnitude $\Phi$ (solar axions) given by Eq. (5) for the solar axions, and the value $\Phi$ (Earth axions) given by (7) for the axions emitted from the Earth's core.

\section{Spectral properties. Results}

We follow the procedure described above in subsection II B and present the axion field in time dependent background as follows

$$
\phi(t, r)=\phi_{w, \delta}\left(r-R_{0}\right)+\chi(t, r)
$$

where $\phi_{w, \delta}\left(r-R_{0}\right)$ satisfies the classical equation of motion while $\chi(t, r)$ describes the time-dependent excitations. As exact solution accounting for the finite size of the nugget is not known we parametrize different simplified solutions by parameter $\delta$. We consider parameter $\delta$ as a probe as explained above.

The next step is to expand the action $\mathcal{S}[\phi]$ by keeping the quadratic terms only,

$\mathcal{S}[\phi]=\mathcal{S}\left[\phi_{w, \delta}\right]+\int d t \int d^{3} x\left[\frac{1}{2} \dot{\chi}^{2}-\frac{1}{2} \chi L_{2}[\delta] \chi\right]+\mathcal{O}\left(\chi^{3}\right)$.

where $L_{2}[\delta]$ is the second order linear differential operator which depends on classical solution $\phi_{w, \delta}\left(r-R_{0}\right)$, and the parameter $\delta$ introduced here is a result of approximation to the true solution $\phi_{w}\left(r-R_{0}\right)$, see Appendix A for the 


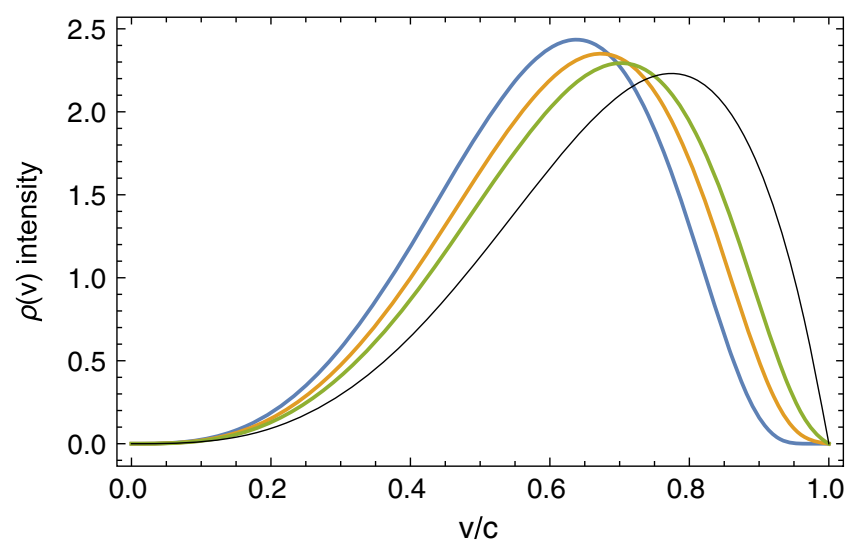

(a) spectrum for $0 \leq v / c \leq 1$

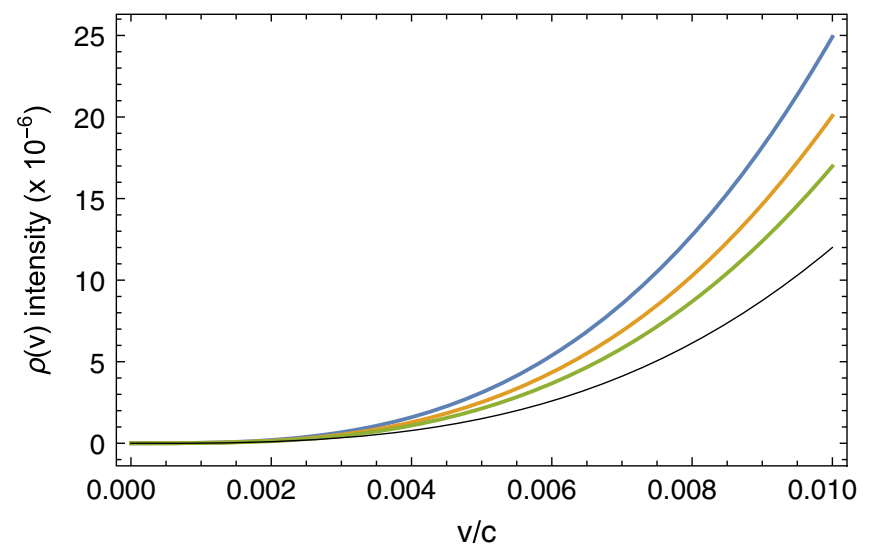

(b) zoom in portion of the spectrum with $0 \leq v / c \leq 0.01$.

FIG. 1. $\rho\left(v_{a}, \delta\right)$ vs $v_{a} / c$. Different values of $\delta$ are chosen respectively: 0 (blue), 0.5 (orange), 1 (green), and 8 (black).

technical details. The next step, as usual, is to expand the fluctuations $\chi$ in terms of complete basis and compute the coefficients $a_{p l m}$ in this expansion. The result for the total radiated energy $E_{\text {rad }}$ is given by Eq. (A24) from Appendix A. It can be presented it in the following form

$$
\begin{aligned}
E_{\mathrm{rad}} & =\int d^{3} x \frac{1}{2} \chi\left[-\frac{\partial^{2}}{\partial t^{2}}+L_{2}[\delta]\right] \chi \\
& =\sum_{l m} \int d^{3} p \frac{1}{2} E_{a}\left|a_{p l m}\right|^{2} \\
& =\sum_{l m} \int_{m_{a}}^{\infty} d E_{a} \cdot 2 \pi p E_{a}^{2}\left|a_{p l m}\right|^{2},
\end{aligned}
$$

where the coefficients $a_{p l m}$ can be explicitly computed and are given by (A22). The expression for the radiated energy (12) allows us to compute the desired spectrum $\rho\left(v_{a}\right)$ defined by (9). The results of the computations are presented on Fig. 1(a) with three different choices of parameter: $\delta=0,0.5$, and 1 for physically realistic conditions, see Appendix A for the details. The low energy portion of the spectrum with $0 \leq v / c \leq 0.01$ is shown on Fig. 1(b).

A few comments are in order. Parameter $\delta$ in our treatment of the problem was introduced as a probe to test our computational scheme which requires to compute all the modes in the background of the classical solution parametrized by parameter $\delta$. While the classical solution itself can be computed numerically, we need some analytical form to proceed with computations of the modes. Parameter $\delta$ is precisely introduced in order to parametrize this analytical expression entering the differential operator $L_{2}[\delta]$. As mentioned above, the parameter $\delta$ roughly varies from 0 to 1 in physically realistic circumstances. With the purpose of the test we performed the computations for different values of $\delta$ shown in Figs. 1, where we also included the "unphysical value" for parameter $\delta=8$ exclusively for illustrative purposes.

One can explicitly see that the results for the spectrum are not very sensitive to parameter $\delta$. As we discuss below, the crucial factor $\xi$ to be introduced in next section and which enters all final formulas is also not sensitive to parameter $\delta$. To reiterate: the basic qualitative results are not very sensitive to choice of parameter $0 \leq \delta \leq 1$.

One next comment goes as follows. It is very instructive to compare our 3D computations with 1D computations presented in [22]. We had anticipated before the 3D computations have been carried out that the results in the relativistic domain $v_{a} / c \gtrsim 0.5$ should not be drastically modified in comparison with simplified treatment in [22]. We can now confirm that this is indeed the case. At the same time we had expected the drastic modification of the spectrum in the nonrelativistic regime $v_{a} / c \leq 0.01$ which is the subject of the present work. Indeed, the 3D spectrum in this domain behaves as $\rho\left(v_{a}\right) \sim v_{a}^{3}$ as shown in Fig. 1b, in contrast with linear dependence in simplified treatment in Ref. [22]. This difference in behaviour at small $v_{a} / c \ll 1$ can be attributed to the phase volume suppression $\sim d^{3} k$ in 3D case for $\lambda_{a} \gg m_{a}^{-1}$.

\section{GRAVITATIONALLY TRAPPED AXIONS}

In the previous section we computed the portion of the axions which have sufficiently low velocities (below escape velocity) such that they will be orbiting the Sun as long as it exists. This portion of the nonrelativistic axions is extremely tiny as shown on Fig. 1(b). Nevertheless, the effect could be drastically enhanced as we discuss below due to accumulation of these axions during entire life of the solar system, i.e., for $\sim 4.5$ billion of years.

The condition for the axions to be bounded after being radiated is determined by the trapped velocity $v_{\text {trapped }}$, defined as 


$$
\begin{aligned}
& \frac{v_{\odot}^{\text {trapped }}}{c}=\sqrt{\frac{2 G M_{\odot}}{c^{2} R_{\odot}}} \simeq 2 \times 10^{-3}, \\
& \frac{v_{\oplus}^{\text {trapped }}}{c}=\sqrt{\frac{2 G M_{\oplus}}{c^{2} R_{\oplus}}} \simeq 3.7 \times 10^{-5},
\end{aligned}
$$

such that all axions with $v \leq v_{\odot}^{\text {trapped }}$ will be trapped by the Sun and the axions with $v \leq v_{\oplus}^{\text {trapped }}$ will be trapped by the Earth. The effect of the trapped axions is not new, and discussed previously in the literature [23]. The goal here is to present some numerical estimates for our specific AQN model when the axions which are produced as a result of the annihilation events can be trapped in the solar atmosphere. These estimates will play a key role in our discussions on the discovery potential of these axions.

\section{A. Solar corona background. Nonresonance case}

According to Fig. 1(b) these highly nonrelativistic axions represent a very tiny portion of the produced axions. The energy which is accumulated in the solar atmosphere per unit time as a result of trapping these axions can be estimated as follows

$$
\begin{aligned}
\frac{d E_{\odot}}{d t}(\text { trapped axions }) & \simeq 1.6 \times 10^{27} \cdot \xi \cdot \frac{\mathrm{erg}}{\mathrm{s}} \\
& \simeq 10^{17} \cdot\left(\frac{\xi}{10^{-10}}\right) \cdot \frac{\mathrm{erg}}{\mathrm{s}}
\end{aligned}
$$

where we used the expression (3) for the rate of the energy transfer to the axions. We also introduced the suppression factor $\xi$ to account for the small fraction of the trapped axions with $v \leq v_{\text {trapped. }}$. For numerical estimates in formula (14) we use suppression factor $\xi \sim 10^{-10}$ computed $^{2}$ in previous section and presented on Fig. 1(b).

The axions (14) could not leave the system during entire life time of the Sun, i.e., 4.5 billion years $\simeq 10^{17} \mathrm{~s}$. Therefore, the total energy accumulated by the Sun and related to AQN annihilation events radiating the slow velocity axions can be estimated as follows

$$
\begin{aligned}
E_{\odot}(\text { trapped axions }) & \simeq 10^{17} \cdot\left(\frac{\xi}{10^{-10}}\right) \cdot \frac{\mathrm{erg}}{\mathrm{s}} \cdot 10^{17} \mathrm{~s} \\
& \simeq 10^{34}\left(\frac{\xi}{10^{-10}}\right) \mathrm{erg} .
\end{aligned}
$$

This energy can be expressed in terms of extra solar mass $\Delta M_{\odot}$ accumulated by the Sun and represented by the trapped axions

\footnotetext{
${ }^{2}$ To demonstrate the insensitivity to parameter $\delta$, we note that $\xi$ shows very moderate changes between $(0.68-1) \times 10^{-10}$ when $\delta$ varies between 0 and 1 . For the "unphysical value" $\delta=8$ the parameter $\xi \simeq 0.48 \times 10^{-10}$, see Appendix A.
}

$$
\Delta M_{\odot}(\text { trapped axions }) \simeq 10^{10}\left(\frac{\xi}{10^{-10}}\right) \mathrm{kg}
$$

which of course represents a very tiny fraction of the solar mass $M_{\odot} \simeq 2 \times 10^{30} \mathrm{~kg}$.

The energy (15) corresponds to the following total number of the axions accumulated by the Sun during its life-time:

$$
\begin{aligned}
N_{\odot}^{\text {axions }} & \sim \frac{E_{\odot}(\text { trapped axions })}{m_{a} c^{2}} \\
& \simeq 10^{51} \cdot\left(\frac{\xi}{10^{-10}}\right) \cdot\left(\frac{10^{-5} \mathrm{eV}}{m_{a}}\right) .
\end{aligned}
$$

If we assume that the majority of these axions are localized within 2 solar radius $R_{\odot}$, we arrive to the following estimate for the average axion energy density inside this volume

$$
\rho_{\odot}^{\text {axions }} \sim \frac{E_{\odot}(\text { trapped axions })}{\frac{4}{3} \pi\left(2 R_{\odot}\right)^{3}} \sim 0.5 \times 10^{3}\left(\frac{\xi}{10^{-10}}\right) \frac{\mathrm{GeV}}{\mathrm{cm}^{3}},
$$

which is 3 orders of magnitude larger than the present average dark matter density today $\rho_{\mathrm{DM}} \simeq 0.3 \frac{\mathrm{GeV}}{\mathrm{cm}^{3}}$. One should comment here that this enhancement of the DM density in the vicinity of the Sun obviously not in contradiction with most precise observational upper limits on solar system (SS) -bound DM, which is normally expressed as $\rho_{\mathrm{SS}}<2 \times 10^{5} \frac{\mathrm{GeV}}{\mathrm{cm}^{3}}$, see, e.g., [30]. It is also interesting to note that some authors [31,32] previously argued that the DM in the SS might be greatly enhanced (on the level of $10^{3}$ ) as a result of capturing of DM particles from the Galactic halo due to the 3 body interaction (the Sun, a planet, and a DM particle). Other authors $[33,34]$ estimated that the effect of capturing is small. We refer to these original papers for the discussions and details. The only comment we would like to make here is that the effect estimated in Eq. (18) is fundamentally distinct in nature in comparison with previously discussed effect [31-34]. The novel effect which is the subject of this work is entirely rooted to the AQN model when the nuggets get disintegrated when enter the solar atmosphere. The corresponding annihilation events produce the low velocities axions with $v \leq v_{\odot}^{\text {trapped }}$. These axions which behave as DM particles surrounding the Sun have no relation to the effect discussed in [31-34].

Now we want to estimate the number density $n_{\odot}^{\text {axions }}$ of these axions assuming, as before, that the majority of the axions are localized within 2 solar radius $R_{\odot}$. 
$n_{\odot}^{\text {axions }}=\frac{N_{\odot}^{\text {axions }}}{\frac{4}{3} \pi\left(2 R_{\odot}\right)^{3}} \simeq 0.5 \times 10^{17}\left(\frac{\xi}{10^{-10}}\right) \cdot\left(\frac{10^{-5} \mathrm{eV}}{m_{a}}\right) \frac{1}{\mathrm{~cm}^{3}}$.

Can these axions be observed? These axions cannot decay as the axion life time $\tau(a \rightarrow 2 \gamma)$ is very long. However, these axions can be converted to photons in the background of external magnetic field. The corresponding probability of this conversion is determined by the formula $[35,36]$ :

$$
\begin{aligned}
P_{a \rightarrow \gamma} & =\sum_{q=q_{ \pm}}\left(\frac{g_{a \gamma} \mathcal{B}}{q}\right)^{2} \sin ^{2}\left(\frac{q L}{2}\right), \\
q_{ \pm} & = \pm \omega-\sqrt{\omega^{2}-m_{a}^{2}}
\end{aligned}
$$

where $L$ is a typical distance where the magnetic field $\mathcal{B}$ is present. For nonrelativistic axions one can approximate $q_{ \pm} \simeq \pm \omega$. Furthermore, for our present analysis we assume that typical $\mathcal{B} \sim 300 \mathrm{G}$ in the solar atmosphere, while $L$ is very large $e^{3}$ such that $\sin ^{2}\left(\frac{q L}{2}\right)$ can be approximated as $\frac{1}{2}$. Therefore, probability of the conversion can be approximated as follows

$$
\begin{aligned}
P_{a \rightarrow \gamma} & \simeq\left(\frac{g_{a \gamma} \mathcal{B}}{m_{a}}\right)^{2} \text { where } \\
\frac{g_{a \gamma}}{m_{a}} & \simeq \frac{\alpha}{2 \pi\left(m_{\pi} f_{\pi}\right)} \cdot\left(\frac{E}{N}-\frac{24+z}{3} \frac{1+z}{1+z}\right) \frac{1+z}{\sqrt{z}},
\end{aligned}
$$

where $z=m_{u} / m_{d} \simeq 0.56$ and parameter $E / N=0$ for KSVZ model, and $E / N=8 / 3$ for DFSZ model. For simple numerical analysis we take $E / N=0$ to arrive to the following estimate

$$
P_{a \rightarrow \gamma} \simeq\left(\frac{g_{a \gamma} \mathcal{B}}{m_{a}}\right)^{2} \sim 10^{-35}\left(\frac{\mathcal{B}}{300 G}\right)^{2}
$$

The number of the produced photons (as a result of the conversion from the axions) per unit volume with the frequency $\omega=m_{a}$ is estimated as follows

$$
\begin{aligned}
\frac{d N(a \rightarrow \gamma)}{d V} & \simeq n_{\odot}^{\text {axions }} \cdot P_{a \rightarrow \gamma} \\
& \simeq 10^{-18}\left(\frac{\mathcal{B}}{300 G}\right)^{2}\left(\frac{\xi}{10^{-10}}\right)\left(\frac{10^{-5} \mathrm{eV}}{m_{a}}\right) \frac{1}{\mathrm{~cm}^{3}}
\end{aligned}
$$

\footnotetext{
${ }^{3} \mathrm{~A}$ rough estimate in the following subsection suggests that $L m_{a} \sim 10^{3}$ if the resonance condition is satisfied, see Eq. (33). For more general nonresonant case a typical length scale is even larger within the classical axion windows, $10^{-6} \mathrm{eV}<m_{a}<$ $10^{-3} \mathrm{eV}$ because there is no requirement for the variation of the plasma frequency on scale $L$ to be small.
}

where $n_{\odot}^{\text {axions }}$ is estimated in (19). These converted photons obviously can leave the system. The total number of photons leaving the system through area $\sim 4 \pi\left(2 R_{\odot}\right)^{2}$ per unit time is given by

$$
\begin{aligned}
\frac{d \Phi(a \rightarrow \gamma)}{d t} & =\frac{d N(a \rightarrow \gamma)}{d V}\left[4 \pi\left(2 R_{\odot}\right)^{2}\right] c \\
& \simeq 10^{16}\left(\frac{\mathcal{B}}{300 G}\right)^{2}\left(\frac{\xi}{10^{-10}}\right)\left(\frac{10^{-5} \mathrm{eV}}{m_{a}}\right) \frac{1}{\mathrm{~s}}
\end{aligned}
$$

These photons are very monochromatic with $\omega=m_{a}$ with accuracy of order $10^{-3}$. Potentially, it gives us some chance to observe them on Earth. The corresponding count of photons $d F(a \rightarrow \gamma)$ arriving from the Sun with monochromatic frequency $\omega=m_{a}$ (due to the axion-photon conversion) is estimated as

$$
\begin{aligned}
\frac{d F(a \rightarrow \gamma)}{d A \cdot d t} & \sim \frac{d \Phi(a \rightarrow \gamma) / d t}{4 \pi D_{\odot}^{2}} \\
& \sim 10^{-12}\left(\frac{\mathcal{B}}{300 \mathrm{G}}\right)^{2}\left(\frac{\xi}{10^{-10}}\right)\left(\frac{10^{-5} \mathrm{eV}}{m_{a}}\right) \frac{1}{\mathrm{~cm}^{2} \cdot \mathrm{s}} .
\end{aligned}
$$

This count, of course, is extremely low. However, these estimates were based on rate (22) corresponding $a \rightarrow \gamma$ conversion in vacuum. As it is known since [35] the rate could be drastically enhanced if the system is placed in a media with non-vanishing plasma frequency $\omega_{p}$ exactly matching the axion mass, i.e., $\omega_{p}=m_{a}$, which represents the topic for the next subsection.

\section{B. Solar corona background. Resonance conversion in solar plasma}

We start with numerical estimation for the plasma frequency $\omega_{p}$ in the solar corona where the most axions are released as a result of the AQN's annihilation events,

$$
\omega_{p} \equiv \sqrt{\frac{4 \pi \alpha n}{m_{e}}} \simeq 3.5 \times 10^{-6} \cdot\left(\frac{n}{10^{10} \mathrm{~cm}^{-3}}\right)^{\frac{1}{2}} \mathrm{eV} .
$$

The numerical similarity between $\omega_{p}$ and the expected value for the axion mass $m_{a}$ from allowed window $m_{a} \in$ $\left(10^{-6}-10^{-3}\right) \mathrm{eV}$ represents the basic motivation for analysis in this subsection. In other words, our goal here is to study possible observational consequences of the resonance case when the condition $\omega_{p}=m_{a}$ could occur in the corona, which is explicit manifestation of the so-called "level-crossing effect" as formulated in Ref. [35].

If the condition $\omega_{p}=m_{a}$ is fulfilled the corresponding resonance $a \rightarrow \gamma$ conversion in media is determined by formula [35]: 


$$
\begin{aligned}
P_{a \rightarrow \gamma} & =\sin ^{2}\left(\Delta_{M} L\right), \quad \Delta_{M}=\frac{\mathcal{B}}{2 M} \sin \theta, \\
M & \equiv g_{a \gamma}^{-1}, \quad \cos \theta \equiv \hat{\overrightarrow{\mathcal{B}}} \cdot \hat{\vec{k}}
\end{aligned}
$$

where we adopted the notations for $\Delta_{M}$ from [35] and expressed the fundamental PQ mass scale $M$ from [35] in terms of the original definition for $g_{a \gamma}$. Of course we do not expect that this condition can be exactly satisfied in reality in nature. Furthermore, the oscillation length $l_{\text {deg }}=\pi / \Delta_{M}$ is very long, much longer than the size of the system such that $P_{a \rightarrow \gamma}$ never becomes of order one effect. However, our goal here is different, and we present formula (27) exclusively for illustrative purposes to illuminate the role of the distance scale where the conversion occurs. With this purpose we expand the resonance expression (27) assuming that $\Delta_{M} L \ll 1$ and compare with nonresonance case (22) to arrive

$$
P_{a \rightarrow \gamma} \simeq\left(\Delta_{M} L\right)^{2} \simeq\left(\frac{g_{a \gamma} \mathcal{B}}{m_{a}}\right)^{2} \cdot\left(\frac{m_{a} L}{2}\right)^{2}
$$

where we consider the special case $\theta=\pi / 2$ to simplify the arguments. Formula (28) illustrates very important point: small conversion rate in nonresonance case (22) corresponds to very short distance $\sim m_{a}^{-1}$ where this conversion takes place. Indeed, the first brackets in (28) identically coincide with formula (22) describing the conversion in nonresonance case. Precisely this first term describes a huge suppression factor.

For our present studies it is important to emphasize that the same formula (28) also explicitly shows that this suppressed conversion (22) can be greatly enhanced with the second factor $\sim\left(m_{a} L\right)^{2}$ if one can increase the coherence length $L$ by maintaining $\omega_{p}=m_{a}$. If the coherence can be maintained on much larger scale than $m_{a}^{-1}$ such that $\left(m_{a} L\right)^{2} \gg 1$ the effect of conversion $P_{a \rightarrow \gamma}$ will be strongly enhanced in comparison with (22) by this large factor $\left(m_{a} L\right)^{2} \gg 1$ entering formula (28). It is clear that one should not expect that the effect could be of order one as one cannot maintain the coherence on the huge scale $l_{\mathrm{deg}}=\pi / \Delta_{M}$. However, some enhancement in comparison with (22) still can be achieved.

The same conclusion also follows from the following expression which was derived using the perturbation theory by treating the inhomogeneities of the magnetic field and plasma density as small perturbations [35]

$$
\begin{aligned}
P_{a \rightarrow \gamma} & =\left|\int_{0}^{L} d z \Delta_{M}(z) \cdot \exp \left(i \Delta_{a} z-i \int_{0}^{z} d z^{\prime} \Delta_{\|}\left(z^{\prime}\right)\right)\right|^{2} \\
\Delta_{a} & =-\frac{m_{a}^{2}}{2 \omega}, \quad \Delta_{\|}=-\frac{\omega_{p}^{2}}{2 \omega}
\end{aligned}
$$

where we neglected $\Delta_{\|}^{\mathrm{vac}} \sim B^{2}$ which is numerically very small for relatively weak typical solar magnetic field.

The conversion rate given by Eq. (29) generates the enhancement proportional to the large length $L$ if the phases maintain the coherence and the cancellations between different phases do not occur due to the fast fluctuations. The requirement that the coherence is maintained up to the scale $L$ is determined by the following condition

$$
\left(\Delta_{a} L-\int_{0}^{L} d z^{\prime} \Delta_{\|}\left(z^{\prime}\right)\right) \lesssim \pi
$$

If this condition is fulfilled then the conversion rate given by Eq. (29) reduces to our previous expression (28) with enhancement factor $\sim L^{2}$, i.e.,

$$
P_{a \rightarrow \gamma} \sim\left(\Delta_{M} L\right)^{2} \sim L^{2} .
$$

This supports our previous conclusion that the enhancement factor $\left(m_{a} L\right)^{2}$ is a result of constructive interference. The corresponding length scale $L$ is determined by condition (30).

Now we want to address the following question: What is the typical length scale $L$ where the condition (30) can be satisfied in solar atmosphere? We limit our analysis with the trapped axions which have nonrelativistic velocities with $\omega \simeq m_{a}$ as discussed in previous Sec. III A. These axions are distributed in the entire solar atmosphere. Therefore, there is always an extended region in corona or chromosphere where the electron density $n$ is such that the plasma frequency (26) equals the axion mass, i.e., $m_{a}=\omega_{p}$. The only question remains to be answered is what are the typical length scales where the average value $\langle n\rangle$ for the electron density varies. ${ }^{4}$

To estimate the corresponding scale $L$ we notice that a typical variation of the density (and the plasma frequency) $n$ by factor $\sim 10$ occurs when the altitude changes by $\sim 10^{3} \mathrm{~km}$. Assuming a linear extrapolation (excluding very fast changes in the transition region) one should expect that the variation of the density $\delta n / n \sim 1$ occurs on the scale of order $l_{0} \sim 10^{2} \mathrm{~km}$. This estimate implies that the relative variation (mismatch) of the density on the coherence scale $L$ of the axion/photon oscillation must not exceed $\lambda / L$ to be consistent with (30). In other words, the scale $L$ where coherence (30) can be maintained must satisfy the following condition

\footnotetext{
${ }^{4}$ Local fluctuations of the density always occur as a result of different types of waves, including the sound waves, in plasma. However, it is expected that these oscillations do not change the integral entering (30). In other words, we are interested in steady and sustained variation of the average density $\langle n\rangle$ with latitude and altitude, rather than numerous conventional fluctuations which always occur in hot plasma but do not modify the average magnitude of the integral (30).
} 


$$
L \sim l_{0} \frac{\lambda}{L} \Rightarrow L \sim \sqrt{\lambda l_{0}} \sim 0.4 \times 10^{4} \sqrt{\frac{10^{-5} \mathrm{eV}}{m_{a}}} \mathrm{~cm} .
$$

Precisely at this coherence scale $L$ the mismatch in plasma frequency is sufficiently small as $(\delta n / n)_{L} \sim \lambda / L$. At the same time $(\delta n / n)_{l_{0}} \sim(\lambda / L) \cdot\left(l_{0} / L\right) \sim 1$ becomes order of one at much larger scales $\sim l_{0}$ where coherence, of course, cannot be maintained. One should emphasize that this very rough estimate assumes a linear extrapolation. This assumption may or may not be justified in reality in solar atmosphere. One should emphasize here that any variations of the magnetic field entering (29) do not modify our estimate for the coherence length (32). This is because the estimate (32) is sensitive to the phase variation [rather than to the amplitude changes $\sim \Delta_{M}(z)$ ] determined by a steady and systematic variation of the plasma frequency $\omega_{p} \sim \sqrt{n}$ in corona.

If one literally accepts the estimate (32) the corresponding enhancement factor can be approximated as follows,

$$
\left(L m_{a}\right)^{2} \sim 4 \times 10^{6}\left(\frac{m_{a}}{10^{-5} \mathrm{eV}}\right) \gg 1 .
$$

Our previous (nonresonance) estimate (25) should be multiplied by the enhancement factor (33) for case if the resonance conditions can be satisfied and the linear extrapolation is justified. The rate of conversion with this factor becomes

$$
\left.\frac{d F(a \rightarrow \gamma)}{d A \cdot d t}\right|_{\text {resonance }} \approx 10^{-6}\left(\frac{\mathcal{B}}{300 \mathrm{G}}\right)^{2}\left(\frac{\xi}{10^{-10}}\right) \frac{1}{\mathrm{~cm}^{2} \cdot \mathrm{s}} .
$$

The corresponding energy flux can be estimated as

$$
\begin{aligned}
m_{a} & \left.\frac{d F(a \rightarrow \gamma)}{d A \cdot d t}\right|_{\text {resonance }} \\
& \approx 10^{-30}\left(\frac{\mathcal{B}}{300 \mathrm{G}}\right)^{2}\left(\frac{\xi}{10^{-10}}\right)\left(\frac{m_{a}}{10^{-5} \mathrm{eV}}\right) \frac{W}{\mathrm{~cm}^{2}},
\end{aligned}
$$

where we expressed the intensity in conventional $\left(W / \mathrm{cm}^{2}\right)$ units using the relations $1 W=10^{7}(\mathrm{erg} / \mathrm{s})$ and $1 \mathrm{eV}=1.6 \times 10^{-12} \mathrm{erg}$.

While the count (34), (35) is still very low, some hope is that this is an unique monochromatic line. Furthermore, the intensity of this line must be correlated with the EUV emission from corona. In addition, during the flares the magnetic field $\mathcal{B}$ might be very large in the solar system which provides some enhancement factor and possible correlations with the flares. Finally, this monochromatic line can be, in principle, discriminated from the background noise as it should appear only along the line-of-sight in the direction of the Sun.
It is very instructive to compare the intensity (35) with corresponding conventional energy flux from the Sun in the frequency band $\omega \approx m_{a}$. To proceed with the estimates we recall that the total solar intensity (integrated over all frequency bands) at Earth surface is about $0.14 \cdot \frac{W}{\mathrm{~cm}^{2}}$, which of course many orders of magnitude higher than the rate (35). However, we should compare (35) not with the total intensity from the Sun measured on Earth, but rather with the solar energy flux from the low energy frequency band with $\omega \leq m_{a}$. The corresponding estimates can be easily performed as the corresponding spectral properties are determined by the Reyleigh-Jeans formula for low energy tail of the black-body $(\mathrm{BB})$ radiation, i.e.,

$d E_{\omega}=\frac{T}{\pi^{2}} \omega^{2} d \omega, \quad E_{\mathrm{tot}}^{\mathrm{BB}}=\frac{\pi^{2}}{15} T^{4}, \quad \frac{E_{\omega \leq m_{a}}}{E_{\mathrm{tot}}^{\mathrm{BB}}} \sim \frac{5}{\pi^{4}}\left(\frac{m_{a}}{T}\right)^{3}$.

The intensity of the conventional BB solar radiation in the low energy frequency band with $\omega \leq m_{a}$ is estimated as follows

$$
\left(0.14 \cdot \frac{W}{\mathrm{~cm}^{2}}\right) \cdot \frac{5}{\pi^{4}}\left(\frac{m_{a}}{T}\right)^{3} \sim 0.4 \times 10^{-16}\left(\frac{m_{a}}{10^{-5} \mathrm{eV}}\right)^{3} \frac{W}{\mathrm{~cm}^{2}},
$$

which is still much higher than the energy flux due to the axion conversion (35) within conventional axion mass window. Only for ultra light axions with $m_{a} \leq 10^{-12} \mathrm{eV}$ the background (37) becomes below the signal (35). The corresponding case of ultralight axion is not part of this work and shall not be further elaborated.

Therefore, one should completely remove the emission from the photosphere with large background (37) for analyzing of the axion conversion (35) from corona. Such removing occurs naturally during the solar eclipses. In practice, astronomers in the past have developed a number of technical tools which allowed to study a weak emission from corona by removing a much stronger radiation from photosphere. A high resolution instrument would be very beneficial to study the monochromatic line (35) with the width $\Delta \omega$, which is determined by the escape velocity $v_{\odot}^{\text {trapped }}$ from (13), i.e., $\Delta \omega \sim m_{a} v_{\odot}^{\text {trapped }} \sim 10^{-3} m_{a}$.

To recapitulate: the expected signal from the solar corona is very low as our estimate (35) suggests. It can be only studied if the background radiation (37) from the photosphere can be removed from analysis and the instrumental resolution is sufficiently high to study the highly monochromatic emission (with the width $\Delta \omega / \omega \sim 10^{-3}$ ) from the corona. Needless to say that a strong magnetic field in a detector is not required for the observation of these photons on Earth because the axion-photon conversion occurs in the solar atmosphere rather than on Earth. In a sense we use the entire Sun as a one big helioscope where the trapped axions 
have been accumulated during 4.5 billion years and where the axions can be converted to photons in the entire solar atmosphere.

In next subsection we consider much more optimistic case when the axions are trapped by the Earth. As we shall see below, the density of such axions could be the same order of magnitude as the galactic axion density, which is the conventional normalization point for the most presently operational (or under construction, or in stage of design) axion search experiments.

\section{Axions from the Earth's underground}

According to Ref. [22] the axion flux due to the AQN annihilation events in the very deep underground is given by (7). We integrate this rate over entire surface to arrive

$$
\begin{aligned}
\frac{d E_{\oplus}}{d t}(\text { trapped axions }) & \sim 10^{16} \xi_{\oplus} \cdot\left(\frac{\Delta B}{B}\right) \cdot 4 \pi R_{\oplus}^{2} \frac{\mathrm{eV}}{\mathrm{s}} \\
& \sim 10^{18} \cdot\left(\frac{\xi_{\oplus}}{10^{-17}}\right) \cdot\left(\frac{\Delta B}{B}\right) \frac{\mathrm{eV}}{\mathrm{s}}
\end{aligned}
$$

where we used the expression (3) for the rate of the energy transfer to the axions. We also introduced the suppression factor $\xi_{\oplus}$ to account for the small fraction of the trapped axions with $v \leq v_{\text {trapped. }}$. For numerical estimates in formula (14) we use suppression factor $\xi_{\oplus} \sim$ $\xi_{\odot} \cdot\left(v_{\oplus} / v_{\odot}\right)^{4} \sim 10^{-17}$ computed in previous section and given by (13). This is of course a very tiny rate even when $\Delta B / B \sim 1$ as we expect.

The axions (38) could not leave the system during entire life time of the Earth, i.e., 4.5 billion years $\simeq 10^{17} \mathrm{~s}$. Therefore, the total energy accumulated by the Earth and related to AQN annihilation events radiating the slow velocity axions can be estimated as follows

$$
\begin{aligned}
E_{\oplus}(\text { trapped axions }) & \simeq 10^{18} \cdot\left(\frac{\xi_{\oplus}}{10^{-17}}\right)\left(\frac{\Delta B}{B}\right) \cdot \frac{\mathrm{eV}}{\mathrm{s}} \cdot 10^{17} \mathrm{~s} \\
& \simeq 10^{35}\left(\frac{\xi_{\oplus}}{10^{-17}}\right)\left(\frac{\Delta B}{B}\right) \mathrm{eV} .
\end{aligned}
$$

This energy can be expressed in terms of extra Earth's mass $\Delta M_{\oplus}$ accumulated by the Earth and represented by the trapped axions

$$
\Delta M_{\oplus}(\text { trapped axions }) \simeq 0.1\left(\frac{\xi_{\oplus}}{10^{-17}}\right)\left(\frac{\Delta B}{B}\right) \mathrm{kg},
$$

which of course represents a very tiny fraction of the Earth mass $M_{\oplus} \simeq 5.9 \times 10^{24} \mathrm{~kg}$.

The energy (39) corresponds to the following total number of the axions accumulated by the Earth during its lifetime:

$$
\begin{aligned}
N_{\oplus}^{\text {axions }} & \sim \frac{E_{\oplus}(\text { trapped axions })}{m_{a} c^{2}} \\
& \simeq 10^{40} \cdot\left(\frac{\xi_{\oplus}}{10^{-17}}\right)\left(\frac{\Delta B}{B}\right) \cdot\left(\frac{10^{-5} \mathrm{eV}}{m_{a}}\right) .
\end{aligned}
$$

If we assume that the majority of these axions are localized within radius $R_{\oplus}$, we arrive to the following estimate for the average axion energy density inside this volume

$\rho_{\oplus}^{\text {axions }} \sim \frac{E_{\oplus}(\text { trapped axions })}{\frac{4}{3} \pi R_{\oplus}^{3}} \sim 0.1\left(\frac{\xi_{\oplus}}{10^{-17}}\right)\left(\frac{\Delta B}{B}\right) \frac{\mathrm{GeV}}{\mathrm{cm}^{3}}$.

which is amazingly close to the average dark matter density today $\rho_{\mathrm{DM}} \simeq 0.3 \frac{\mathrm{GeV}}{\mathrm{cm}^{3}}$. The Eq. (42) should be viewed as the order of magnitude estimate at the very best. The main uncertainty here is that the trapped axions are not distributed uniformly, as assumed in (42). Instead, they are obviously distributed in a highly nontrivial way determined by the position of the nugget when emission occurs (in deep underground) and the direction of the velocity at the moment of emission. Though the estimate (42) is rough, it is also very promising as it suggests that the galactic axion density and the axion density produced by the AQN mechanism could be the same order of magnitude.

One may wonder ${ }^{5}$ if the terrestrial geomagnetic field can be used as the axion converter, and if the resonance conversion may occur on Earth, similar to our discussions in previous Sec. III B devoted to the resonance conversion in solar corona. Unfortunately, for the conventional axion mass window $m_{a} \in\left(10^{-6}-10^{-3}\right) \mathrm{eV}$ the resonance conditions cannot be satisfied. Indeed, while the Earth's ionosphere is highly ionized, the corresponding electron density is very low: $n \sim 10^{6} \mathrm{~cm}^{-3}$ for the so-called F-layer which extends from the altitude $150 \mathrm{~km}$ for few hundred kilometers. The corresponding plasma frequency in the ionosphere

$$
\omega_{p} \equiv \sqrt{\frac{4 \pi \alpha n}{m_{e}}} \simeq 3.5 \times 10^{-8} \cdot\left(\frac{n}{10^{6} \mathrm{~cm}^{-3}}\right)^{\frac{1}{2}} \mathrm{eV}
$$

is well below the typical axion mass. This estimate suggests that the resonance case cannot be realized for the conventional axion mass window. Therefore, one should use the nonresonance formula for conversion:

$$
\begin{aligned}
m_{a} \frac{d F_{\oplus}(a \rightarrow \gamma)}{d A \cdot d t} & \simeq \frac{1}{2} \rho_{\oplus}^{\text {axions }} P_{a \rightarrow \gamma} c \\
& \simeq 10^{-41}\left(\frac{\xi_{\oplus}}{10^{-17}}\right)\left(\frac{\Delta B}{B}\right)\left(\frac{\mathcal{B}}{0.5 G}\right)^{2} \frac{W}{\mathrm{~cm}^{2}} .
\end{aligned}
$$

\footnotetext{
${ }^{5}$ We are thankful to anonymous Referee who suggested that we produce such estimates.
} 
This estimate indicates that the corresponding rate is too low to be observed if one uses the terrestrial geomagnetic field as the axion converter. The crucial suppression factors here are the small terrestrial geomagnetic magnetic field $\mathcal{B} \sim 0.5 \mathrm{G}$ and very small escape velocity (13) which leads to the very tiny portion of the trapped axions $\xi_{\oplus} \sim 10^{-17}$. Similar arguments also apply to other planets such as Jupiter, which has no resonant enhancement nor sufficiently strong magnetic field comparable to the solar sunspots. Therefore, while the AQNs obviously get annihilated in the Jupiter's underground producing additional internal heat, the corresponding axion emission would be even smaller than from the Sun (35) due to a number of additional suppression factors such as smaller mass (and therefore, smaller impact parameter leading to a smaller AQN flux hitting Jupiter), larger distance from Earth, smaller escape velocity (leading to a smaller parameter $\xi$ ) in comparison with the solar $\xi_{\odot}$, etc.

Therefore we return to our main and most promising estimate (42) which indicates that the density of the bound axions could be the same order of magnitude as the galactic DM axions, and therefore the conventional instruments originally designed for the galactic axion searches can be also used to study the trapped axions. The distinct feature of the AQN trapped axions is very large wave length $\lambda_{a}=$ $\left(m_{a} v_{a}\right)^{-1}$ as the typical trapped velocity $v_{a} \leq v_{\oplus}^{\text {trapped }}$ is much smaller than a typical galactic DM velocity $\sim 10^{-3} c$ according to (13). This unique feature of the trapped axions might be the "smoking gun" leading to their discovery.

\section{CONCLUSION AND FUTURE DIRECTIONS}

This work represents a natural generalization of the previous studies [22] to properly account for the production of the low energy axions when the AQNs get annihilated in the Sun or Earth and emit axions with $v \leq v_{\odot}^{\text {trapped }}$ in the solar corona or $v \leq v_{\oplus}^{\text {trapped }}$ in the deep Earth's underground. This portion of the nonrelativistic axions is extremely tiny as shown on Fig. 1(b). However, the effect is drastically enhanced as argued in Sec. III due to accumulation of these axions during entire life of the solar system, i.e., for $\sim 4.5$ billion of years. The corresponding estimates represent the main results of the present studies.

We shall not repeat and discuss here a large number of estimates presented in Sec. III. Instead, we focus on a single formula (42) describing the energy density of the trapped axions $\rho_{\oplus}^{\text {axions. }}$. We think this estimate has a huge discovery potential because $\rho_{\oplus}^{\text {axions }}$ is relatively large and comparable with the average galactic dark matter density today $\rho_{\mathrm{DM}} \simeq 0.3 \frac{\mathrm{GeV}}{\mathrm{cm}^{3}}$. What is more important is that the spectral features of the trapped axions are very distinct from conventional galactic axions because the typical trapped velocity $v_{a} \leq v_{\oplus}^{\text {trapped }}$ is much smaller than a typical galactic DM velocity $\sim 10^{-3} c$ according to (13). Therefore, the typical wave length $\lambda_{a}=\left(m_{a} v_{a}\right)^{-1}$ of these axions is much longer in comparison with galactic axions. This unique feature makes the trapped axions are very distinct from conventional galactic axions. These axions obviously can be easily discriminated from anything else. The discovery of such axions would be a "smoking gun" for the entire AQN proposal unifying the DM and baryogenesis (separation of charges) problems.

This new mechanism of the axion production is entirely based on the unorthodox AQN dark matter model. Why do we think that this new AQN framework (and accompanying the axion emission) should be taken seriously? We refer to [22] for overview of this DM model. Nevertheless, we want to make few comments here suggesting that the AQN framework should be indeed taken seriously.

We start with the remark that this model was invented long ago as a natural explanation of the observed ratio $\Omega_{\text {dark }} \sim \Omega_{\text {visible }}$ between visible and dark matter densities. In context of the present work the most important feature of this model is that it may potentially resolve the old renowned puzzle (since 1939) known in the community under the name "the solar corona mystery." In particular, this model, without adjusting any parameters, generates the observed EUV luminosity (2) as reviewed in [22].

Furthermore, the AQN resolution of the solar corona puzzle also resolves another mystery [37] where it was claimed that a number of highly unusual phenomena observed in solar atmosphere might be related to the gravitational lensing of "invisible" streaming matter towards the Sun which is correlated with positions of the planets. This is really a weird correlation because one should not expect any connections between the flare occurrences, the intensity of the EUV radiation, and the position of the planets. At the same time, such "weird" correlations naturally occur within AQN framework. This is because the dark matter AQNs, being the "invisible streaming matter" (in terminology of Ref. [37]) can play the role of the triggers sparking the large flares [38]. Therefore, the observation of the correlation between the EUV intensity, the frequency of the flares and positions of the planets can be considered as an additional supporting argument of the dark matter explanation of the observed EUV irradiation (2), because both effects are originated from the same dark matter AQNs.

Last, but not least. The AQN model offers a very natural resolution of the so-called "primordial lithium puzzle" as recently argued in [39]. This problem has been with us for at least two decades, and conventional astrophysical and nuclear physics proposals could not resolve this longstanding mystery. In the AQN framework this puzzle is automatically and naturally resolved without adjusting any parameters as shown in [39]. This resolution represents yet another, though indirect, support for this new AQN framework.

All these arguments obviously represent indirect support for the AQN paradigm. The discovery of the trapped axions 
with energy density (42) and with drastically distinct spectral features (in comparison with conventional galactic axions) would be the direct support for this model as it is hard to imagine any other model which could produce the axions with $v_{a} \leq v_{\oplus}^{\text {trapped }}$ with sharp cutoff in density for $v_{a}>v_{\oplus}^{\text {trapped }}$. We conclude this work on this optimistic note.

\section{ACKNOWLEDGMENTS}

One of us (A.Z.) is thankful to Konstantin Zioutas for enormous number of questions which motivated these studies. We are thankful to Ludo van Waerbeke for discussions on feasibility and perspective to drastically improve the order of magnitude estimates (18), (42) using numerical Monte Carlo simulations accounting for all possible axion orbits. This research was supported in part by the Natural Sciences and Engineering Research Council of Canada.

\section{APPENDIX A: TECHNICAL DETAILS. AXION EMISSION FROM THE DOMAIN WALL. 3D CASE}

In this Appendix we want to study the spectral properties of the axion's emission as a result of time-dependent perturbations of the axion domain wall. We want to focus on the axion portion of the axion DW, which also includes other fields such as $\pi, \eta^{\prime}$, see [40]. It also contains a phase describing the baryon charge distribution on the surface of the nugget as discussed in [25]. Exact features of the profile functions for all these fields are not important for our purposes. Therefore, one can simplify our computations by considering the following effective Lagrangian with two degenerate vacuum states. ${ }^{6}$

$$
\mathcal{S}[\phi]=\int d^{4} x\left[\frac{1}{2}\left(\partial_{\mu} \phi\right)^{2}-\frac{g^{2}}{4}\left(\phi^{2}-\frac{\pi^{2}}{4} f_{a}^{2}\right)^{2}\right],
$$

where $g=\frac{\sqrt{2}}{\pi} \frac{m_{a}}{f_{a}}$, and we set the effective axion angle as $\phi / f_{a} \equiv \theta+\arg \operatorname{det} M+\pi / 2$ (note that we shift the angle by $\pi / 2$ for convenience of calculation). In this work, we are especially interested in the nonrelativistic domain where thin-wall approximation is badly violated. Thus, we should approach the solution in 3D case. Since the ground-state

\footnotetext{
${ }^{6}$ In our previous studies [25-27] we always discussed the socalled $N=1$ domain walls. It implies that the vacuum is unique and the DW solution interpolates between one and the same physical vacuum. This interpolation always occurs as a result of variation of the axion field together with another fields, such as $\pi$ or $\eta^{\prime}$ as discussed in [40]. These additional fields do not generate much changes in the domain wall tension, nor they affect our analysis of the axion production, which is the subject of the present work. Therefore, we ignore these fields to simplify notations and qualitative analysis in this work.
}

domain wall solution must preserve spherical symmetry, the equation of motion reads

$$
\begin{aligned}
\frac{\partial^{2}}{\partial r^{2}} \phi(r)+\frac{2}{r} \frac{\partial}{\partial r} \phi(r) & =g^{2} \phi(r) \cdot\left[\phi^{2}(r)-\frac{\pi^{2}}{4} f_{a}^{2}\right], \\
\phi\left(R_{0}\right) & =0
\end{aligned}
$$

where $R_{0}$ defines the boundary which separates two distinct physical vacua and it coincides with the radius of the AQN in equilibrium. While the exact domain wall solution to Eq. (A2) is hard to solve, the approximate solution gives ${ }^{7}$

$\phi_{w, R_{0}}(r) \simeq \begin{cases}\frac{\pi}{2} f_{a} \cdot \frac{R_{\mathrm{eff}}}{r} \tanh \left[\frac{1}{2} m_{a}\left(r-R_{0}\right)\right], & R_{0} \lesssim r \leq R_{\text {trans }} \\ \frac{\pi}{2} f_{a} \cdot \tanh \left[\frac{1}{2} m_{a}\left(r-R_{0}+\delta_{R}\right)\right], & r>R_{\text {trans }}\end{cases}$

where $R_{\text {eff }}$ and $\delta_{R}$ are functions of a tunable parameter $R_{\text {trans }}$

$$
\begin{gathered}
\delta_{R} \simeq \frac{1}{R_{0}}\left(R_{\text {trans }}-R_{0}\right)^{2} \equiv \frac{1}{m_{a}} \delta, \\
R_{\text {eff }} \simeq R_{\text {trans }} \frac{\tanh \left[\frac{1}{2 R_{0}} m_{a} R_{\text {trans }}^{2}\right]}{\tanh \left[\frac{1}{2} m_{a} R_{\text {trans }}\right]}
\end{gathered}
$$

within domain $R_{0}<R_{\text {trans }} \lesssim \sqrt{m_{a} R_{0}} \cdot m_{a}^{-1}$. One can explicitly check this approximate solution (A3) is continuous and first order differentiable. Also, it is precisely the exact solution in the near-field limit $r \sim R_{0}$ and the far-field limit $r \gg m_{a}^{-1}$. Hence, the only unknown part the solution is the "transition" regime between these two limits, where we introduce a tunable parameter $R_{\text {trans }}$ to account for this type of error source. We will keep this parameter in the following calculations, so it serves as a probe to test whether the final result is sensitive to our crude approach in the transition regime. As we will see, the final result is not sensitive to the tuning of $R_{\text {trans }}$.

Lastly, instead of using $R_{\text {trans }}$ directly, it is more convenient to define a simple parameter $\delta \equiv m_{a} \delta_{R}$ which roughly varies from 0 to 1 . As we will see, $\delta$ is the only parameter entering the final result.

We are now ready to compute the excitations $\chi(t, z)$ in the time dependent background. These excitations will be eventually identified as the axions emitted by the axions DW. To achieve this task we expand $\phi(t, z)=$ $\phi_{w}\left(z-R_{0}\right)+\chi(t, z)$, which gives

$$
\mathcal{S}[\phi]=\mathcal{S}\left[\phi_{w}\right]+\int d t \int d^{3} x\left[\frac{1}{2} \dot{\chi}^{2}-\frac{1}{2} \chi L_{2} \chi\right]+\mathcal{O}\left(\chi^{3}\right) .
$$

\footnotetext{
${ }^{7}$ Note that interaction between axion and other fields such as $\pi$ and $\eta^{\prime}$ becomes strong within $r \lesssim R_{0}$, see [40]. Hence, we should set a cutoff range at $r \lesssim R_{0}$ where Eq. (A2) is no longer valid.
} 
where $L_{2}$ is a linear differential operator of the second order (with $v \equiv \frac{\pi}{2} f_{a}$ ),

$$
\begin{aligned}
L_{2} \chi= & -\frac{1}{r} \frac{\partial^{2}(r \chi)}{\partial r^{2}}-\frac{1}{r^{2}}\left[\frac{1}{\sin \theta} \frac{\partial}{\partial \theta}\left(\sin \theta \frac{\partial \chi}{\partial \theta}\right)+\frac{1}{\sin ^{2} \theta} \frac{\partial^{2} \chi}{\partial \phi^{2}}\right] \\
& +\left.\left[2 g^{2} \phi^{2} \chi+g^{2}\left(\phi^{2}-v^{2}\right) \chi\right]\right|_{\phi=\phi_{w, R_{0}}} \\
= & -\frac{1}{r} \frac{\partial^{2}(r \chi)}{\partial r^{2}}-\frac{1}{r^{2}}\left[\frac{1}{\sin \theta} \frac{\partial}{\partial \theta}\left(\sin \theta \frac{\partial \chi}{\partial \theta}\right)+\frac{1}{\sin ^{2} \theta} \frac{\partial^{2} \chi}{\partial \phi^{2}}\right] \\
& +\frac{1}{2} \frac{m_{a}^{2}}{v^{2}}\left(3 \phi_{w, R_{0}}^{2}-v^{2}\right) \chi .
\end{aligned}
$$

The corresponding equation of motion is therefore

$$
\frac{\partial^{2}}{\partial t^{2}} \chi=-L_{2} \chi
$$

To look for the initial conditions, we now want to describe the emission of axions in one cycle of oscillation. As mentioned in Sec. II B, annihilation of baryon charge results in oscillations of domain wall. Assuming the oscillation is approximately adiabatic, it is sufficient to only analyze the first half of an oscillation-say, the "contraction period"- where the domain wall shrinks from $R_{0}$ to a slightly smaller size $R_{0}-\Delta R$. We assumed the rest half of the cycle, the "expansion period," is just the timereversed and produces an equivalent contribution. We may write down such initial conditions as

$$
\begin{aligned}
\phi(0, r) & =\phi_{w, R_{0}}(r) \\
\phi\left(\frac{1}{2} t_{\mathrm{osc}}, r\right) & =\phi_{w, R_{0}-\Delta R}(r)+(\text { excitations })
\end{aligned}
$$

where $t_{\mathrm{osc}}$ denotes the period of one full oscillation. The excitation modes in condition (A8b) is unknown and depends on the conversion rate from excitation modes to freely propagating axions. In terms of $\chi$, the initial conditions (A8) imply

$$
\begin{aligned}
& \chi(0, r)=0 \\
& \chi(0, r)=\eta(\theta, \varphi) \partial_{R_{0}}\left[\phi_{w, R_{0}}(r)\right] \Delta R+\mathcal{O}\left(\Delta R^{2}\right)
\end{aligned}
$$

where we introduce a free parameter $\eta(\theta, \varphi)$ which may be interpreted as the "amplitude of efficiency" of the conversion rate from excitations to free axions, so $\eta$ must vary between 0 to 1 . However, $\eta$ here may be also interpreted as a correction term like $\delta$ in the approximate solution (A3) within the transition regime $R_{0} \ll r \lesssim m_{a}^{-1}$, so $\eta$ can be greater than 1 in general. Nonetheless, we will expect $\eta \sim 1$ and will treat it as a normalization factor regarding to the luminosity. And in general, $\eta$ can be expanded by partial waves

$$
\begin{aligned}
\eta(\theta, \varphi) & =\sum_{l=0}^{\infty} \sum_{m=-l}^{l} \eta_{l m} Y_{l m}(\theta, \varphi), \\
\eta_{l m} & =\int_{0}^{2 \pi} d \varphi \int_{0}^{\pi} d \theta \sin \theta Y_{l m}^{*}(\theta, \varphi) \eta(\theta, \varphi) .
\end{aligned}
$$

If we assume a good spherical symmetry preserves during the most period of the annihilation process of AQN, then $\eta_{00}$ will be the dominant contribution and $\eta_{10}$ be the next order correction.

To solve for the excitation mode, it is convenient to write $\chi$ in terms of some normalized basis. The expansion for free wave is conventionally

$$
\begin{aligned}
\chi(t, r, \theta, \varphi) & =\sum_{l=0}^{\infty} \sum_{m=-l}^{l} \int d^{3} p a_{p l m}(t) \chi_{p l m}(r, \theta, \varphi), \\
\chi_{p l m}(r, \theta, \varphi) & =\frac{1}{\sqrt{4 \pi^{2} E_{a}}} j_{l}(p r) Y_{l m}(\theta, \varphi)
\end{aligned}
$$

where $j_{l}(x)$ is the spherical Bessel function, and we have implicitly used two orthogonalities

$$
\begin{aligned}
& \int_{0}^{\infty} d r r^{2} j_{l}(p r) j_{l}(q r)=\frac{\pi}{2 p^{2}} \delta(p-q), \\
& \int_{0}^{2 \pi} d \varphi \int_{0}^{\pi} d \theta \sin \theta Y_{l m}^{*}(\theta, \varphi) Y_{l^{\prime} m^{\prime}}(\theta, \varphi)=\delta_{l l^{\prime}} \delta_{m m^{\prime}} .
\end{aligned}
$$

Note that $L_{2}$ is diagonal in basis of $\chi_{p l m}$

$$
\begin{aligned}
& \int d^{3} x \chi_{q l^{\prime} m^{\prime}}^{*}(r, \theta, \varphi) L_{2} \chi_{p l m}(r, \theta, \varphi) \\
& =\frac{1}{8 \pi E_{a}} \delta(p-q)+\frac{m_{a}^{2}}{4 \pi^{2} E_{a}} K_{p q}^{(l)} \int d r r^{2} j_{l}(q r) j_{l}(p r) \\
& =\frac{\delta(p-q)}{8 \pi E_{a} p^{2}}\left(p^{2}+K_{p, q}^{(l)} m_{a}^{2}\right),
\end{aligned}
$$

where $K_{p, q}^{(l)}$ is a coefficient defined as

$K_{p, q}^{(l)} \equiv \lim _{L \rightarrow \infty} \frac{\int_{0}^{L} d r r^{2} j_{l}(p r) j_{l}(q r) \frac{1}{2}\left[3\left(\frac{1}{v} \phi_{w, R_{0}}(r)\right)^{2}-1\right]}{\int_{0}^{L} d r r^{2} j_{l}(p r) j_{l}(q r)}$

for simplicity of calculation. In B we can show $K_{p, q}^{(l)} \delta(p-q)=\delta(p-q)$. Then Eq. (A7) is simplified to $\frac{d^{2}}{d t^{2}} a_{p l m}(t)=-E_{a}^{2}(p) a_{p l m}(t), \quad E_{a}(p) \equiv \sqrt{p^{2}+m_{a}^{2}}$, 
which clearly has solution

$$
a_{p l m}(t)=b_{p l m} \sin E_{a} t
$$

following the initial condition (A9a), where $b_{p l m}$ is an time-independent coefficient to be determined. To find $b_{p l m}$, we should impose the second initial condition (A9b) which implies

$$
\begin{aligned}
b_{p l m}= & \frac{\pi}{2} \frac{f_{a} m_{a} \Delta R \eta_{l m}}{\sin \left(\frac{1}{2} E_{a} t_{\mathrm{osc}}\right)} \sqrt{\frac{E_{a}}{4 \pi^{2}}}\left\{\int_{0}^{R_{\text {trans }}} d r r^{2} \cdot \frac{R_{\mathrm{eff}}}{r} \operatorname{sech}^{2}\left[\frac{m_{a}}{2}\left(r-R_{0}\right)\right] j_{l}(p r)\right. \\
& -\int_{0}^{R_{\mathrm{trans}}} d r r^{2} \cdot \operatorname{sech}^{2}\left[\frac{m_{a}}{2}\left(r-R_{0}+\delta_{R}\right)\right] j_{l}(p r) \\
& \left.+\int_{0}^{\infty} d r r^{2} \cdot \operatorname{sech}^{2}\left[\frac{m_{a}}{2}\left(r-R_{0}+\delta_{R}\right)\right] j_{l}(p r)\right\} .
\end{aligned}
$$

Note that only the last term in the curly bracket is dominant because $R_{\text {trans }} \ll m_{a}^{-1}$ largely suppresses the first two terms. ${ }^{8}$ Thus, we conclude

$$
b_{p l m} \simeq \frac{\pi}{2} \frac{f_{a} m_{a} \Delta R \eta_{l m}}{\sin \left(\frac{1}{2} E_{a} t_{\mathrm{osc}}\right)} \sqrt{\frac{E_{a}}{4 \pi^{2}}}\left\{\int_{0}^{\infty} d r r^{2} \cdot \operatorname{sech}^{2}\left[\frac{m_{a}}{2}\left(r+\delta_{R}\right)\right] j_{l}(p r)+\mathcal{O}\left(R_{\text {trans }}^{l+2}\right)\right\},
$$

where we have also drop $R_{0}$ in the hyperbolic secant function because it is of order $R_{\text {trans. }}$ This integral can be evaluated precisely if we expand the hyperbolic secant as

$$
\begin{aligned}
\operatorname{sech}^{2}\left(\frac{1}{2} x\right) & =e^{-x} \sum_{n=0}^{\infty}(-1)^{n}(n+1) \frac{1}{2^{n}}\left(e^{-x}-1\right)^{n} \\
& =\sum_{n=0}^{\infty} \sum_{k=0}^{n} \frac{1}{2^{n}} \frac{(n+1) !}{k !(n-k) !}(-1)^{k} e^{-(k+1) x}
\end{aligned}
$$

and use the fact

$$
\begin{aligned}
\int_{0}^{\infty} d \rho \rho^{2} \cdot e^{-(k+1) \rho} j_{l}(p \rho) & =\frac{\sqrt{\pi}}{2^{l+1}} \frac{p^{l}}{(k+1)^{l+3}} \Gamma(l+3) \cdot f\left(\frac{1}{2}(l+3), \frac{1}{2}(l+4), l+\frac{3}{2} ; \frac{-p^{2}}{(k+1)^{2}}\right) \\
f(a, b, c ; z) & \equiv \frac{1}{\Gamma(c)}{ }_{2} F_{1}(a, b, c ; z),
\end{aligned}
$$

where ${ }_{2} F_{1}(a, b, c ; z)$ is the Gauss hypergeometric function, and $f(a, b, c ; z)$ is defined to be the regularized version of ${ }_{2} F_{1}(a, b, c ; z)$ in a conventional way, see Refs. [41,42] and recent article [43]. As discussed in Sec. III, we are especially interested in the nonrelativistic domain, in this limit we have

$$
f\left(\frac{1}{2}(l+3), \frac{1}{2}(l+4), l+\frac{3}{2} ; \frac{-p^{2}}{(k+1)^{2}}\right) \simeq \frac{1}{\Gamma\left(l+\frac{3}{2}\right)}\left[1-\frac{(l+3)(l+4)}{4(k+1)^{2}} \frac{\Gamma\left(l+\frac{3}{2}\right)}{\Gamma\left(l+\frac{5}{2}\right)} p^{2}+\mathcal{O}\left(p^{4}\right)\right]
$$

Combing Eqs. (A16), (A18), (A20), and (A21), we conclude

$$
\begin{aligned}
a_{p l m}(t) & =\eta_{l m} \frac{f_{a} \Delta R e^{-\delta}}{2^{l+3} m_{a}^{2}} \sqrt{\pi E_{a}} \frac{\sin \left(E_{a} t\right)}{\sin \left(\frac{1}{2} E_{a} t_{\mathrm{osc}}\right)} \frac{\Gamma(l+3)}{\Gamma\left(l+\frac{3}{2}\right)}\left(\frac{p}{m_{a}}\right)^{l} H_{l}(p, \delta) \\
& \simeq \eta_{l m} \frac{f_{a} \Delta R e^{-\delta}}{2^{l+3} m_{a}^{2}} \sqrt{\pi E_{a}} \frac{\sin \left(E_{a} t\right)}{\sin \left(\frac{1}{2} E_{a} t_{\mathrm{osc}}\right)} \frac{\Gamma(l+3)}{\Gamma\left(l+\frac{3}{2}\right)}\left(\frac{p}{m_{a}}\right)^{l} H_{l}(0, \delta)\left[1+\mathcal{O}\left(p / m_{a}\right)^{2}\right]
\end{aligned}
$$

\footnotetext{
${ }^{8}$ More specifically, due to the fact $R_{0} \ll m_{a}^{-1}$, we have the hierarchy $R_{0}<R_{\text {trans }} \lesssim \frac{1}{2} \sqrt{m_{a} R_{0}} \cdot m_{a}^{-1} \ll m_{a}^{-1}$.
} 
where we define $H_{l}(p, \delta)$ to be the summation series

$$
H_{l}(p, \delta) \equiv \sum_{n=0}^{\infty} \sum_{k=0}^{n} \frac{e^{-k \delta}}{2^{n}} \frac{(n+1) !}{k !(n-k) !} \frac{(-1)^{k}}{(k+1)^{l+3}} \Gamma\left(l+\frac{3}{2}\right) f\left(\frac{1}{2}(l+3), \frac{1}{2}(l+4), l+\frac{3}{2} ; \frac{-\left(p / m_{a}\right)^{2}}{(k+1)^{2}}\right) .
$$

Then, the total radiation energy $E_{\text {rad }}$ of the domain wall is obviously

$$
E_{\mathrm{rad}}=\int d^{3} x \frac{1}{2} \chi\left[-\frac{\partial^{2}}{\partial t^{2}}+L_{2}\right] \chi=\sum_{l m} \int d^{3} p \frac{1}{2} E_{a}\left|a_{p l m}\right|^{2}=\sum_{l m} \int_{m_{a}}^{\infty} d E_{a} \cdot 2 \pi p E_{a}^{2}\left|a_{p l m}\right|^{2} .
$$

More generally, assuming now the flux is produced within a "cavity of radiation" $V_{\text {rad }}$, the density of radiation energy (per unit volume) is therefore $E_{\text {rad }} / V_{\text {rad. }}$ Then the net flux $\Phi_{\text {rad }}$ going through the boundary of the cavity is clearly

$$
\frac{1}{S_{\mathrm{rad}}} \frac{d}{d E_{a}} \Phi_{\mathrm{rad}}=\frac{p}{E_{a}^{2}} \frac{d}{d E_{a}}\left(\frac{E_{\mathrm{rad}}}{V_{\mathrm{rad}}}\right)=\sum_{l m} \frac{2 \pi p^{2}}{V_{\mathrm{rad}}}\left|a_{p l m}\right|^{2}
$$

Let $R_{\text {rad }} \equiv \frac{V_{\text {rad }}}{S_{\text {rad }}}$ defines the effective size of cavity of radiation, we obtain

$$
\begin{aligned}
\frac{d}{d E_{a}} \Phi_{\mathrm{rad}} & =\sum_{l m} \frac{\eta_{l m}^{2}}{R_{\mathrm{rad}}} \frac{f_{a}^{2} \Delta R^{2}}{m_{a}^{2}} \frac{\pi^{2} e^{-2 \delta}}{2^{2 l+5}}\left[\frac{\Gamma(l+3)}{\Gamma\left(l+\frac{3}{2}\right)}\right]^{2}\left[\frac{\sin \left(E_{a} t\right)}{\sin \left(\frac{1}{2} E_{a} t_{\mathrm{osc}}\right)}\right]^{2} E_{a}\left(\frac{p}{m_{a}}\right)^{2 l+2}\left|H_{l}(p, \delta)\right|^{2} \\
& \simeq \sum_{l m} \frac{\eta_{l m}^{2}}{R_{\mathrm{rad}}} \frac{f_{a}^{2} \Delta R^{2}}{m_{a}^{2}} \frac{\pi^{2} e^{-2 \delta}}{2^{2 l+5}}\left[\frac{\Gamma(l+3)}{\Gamma\left(l+\frac{3}{2}\right)}\right]^{2}\left[\frac{\sin \left(E_{a} t\right)}{\sin \left(\frac{1}{2} E_{a} t_{\mathrm{osc}}\right)}\right]^{2} E_{a}\left(\frac{p}{m_{a}}\right)^{2 l+2}\left|H_{l}(0, \delta)\right|^{2}+\mathcal{O}\left(p / m_{a}\right)^{2 l+4}
\end{aligned}
$$

A few comments should be made regarding to the magnitude of $R_{\text {rad }}$. First, $V_{\text {rad }}$ is defined as the cavity where radiation happens, so $R_{\text {rad }} \simeq \Delta R$ in 1D case where thin-wall approximation is assumed. However, in 3D $R_{\text {rad }}$ may extend to order of $R_{0}$ or even $m_{a}^{-1}$. More generally, it is reasonable to conjecture $R_{\text {rad }}$ can depend on the angular momentum $l$. It is clear that to compute or even estimate the order of $R_{\text {rad }}$ is very difficult. Thus, we should not bother the details of $R_{\text {rad }}$, but rather treat it as a tunable normalization parameter and maybe absorb it into $\eta_{l m}$ if applicable.

We also express the spectra as a function of flux velocity

$$
\begin{aligned}
\frac{d}{d v_{a}} \Phi_{\mathrm{rad}} & =\sum_{l m} \frac{\eta_{l m}^{2}}{R_{\mathrm{rad}}} \frac{f_{a}^{2} \Delta R^{2}}{m_{a}^{3}} \frac{\pi^{2} e^{-2 \delta}}{2^{2 l+5}}\left[\frac{\Gamma(l+3)}{\Gamma\left(l+\frac{3}{2}\right)}\right]^{2}\left[\frac{\sin \left(E_{a} t\right)}{\sin \left(\frac{1}{2} E_{a} t_{\mathrm{osc}}\right)}\right]^{2} E_{a}^{3}\left(\frac{p}{m_{a}}\right)^{2 l+3}\left|H_{l}(p, \delta)\right|^{2} \\
& \simeq \sum_{l m} \frac{\eta_{l m}^{2}}{R_{\mathrm{rad}}} \frac{f_{a}^{2} \Delta R^{2}}{m_{a}^{2}} \frac{\pi^{2} e^{-2 \delta}}{2^{2 l+5}}\left[\frac{\Gamma(l+3)}{\Gamma\left(l+\frac{3}{2}\right)}\right]^{2}\left[\frac{\sin \left(E_{a} t\right)}{\sin \left(\frac{1}{2} E_{a} t_{\mathrm{osc}}\right)}\right]^{2} E_{a}^{3}\left(\frac{p}{m_{a}}\right)^{2 l+3}\left|H_{l}(0, \delta)\right|^{2}+\mathcal{O}\left(p / m_{a}\right)^{2 l+5}
\end{aligned}
$$

If the spherical symmetry is well preserved during most period of the annihilation of $\mathrm{AQNs}$, then $\eta_{00}$ is the dominant term and Eq. (A27) can be simplified considerably. We plot this (normalized) result in Figs. 1. One can see the final result is not very sensitive to the parameter $\delta$. Such spectrum indicates an average energy $\left\langle E_{a}\right\rangle \simeq 1.35 m_{a}$ and an average velocity $\left\langle v_{a}\right\rangle \simeq 0.6 c$. Comparing to the 1D case where $\left\langle E_{a}\right\rangle \simeq 1.18 m_{a}$ and $\left\langle v_{a}\right\rangle \simeq 0.5 c$ [22], we conclude that the general features of the spectrum in the relativistic regime for $v_{a} \geq 0.5 c$ is qualitatively consistent between $1 \mathrm{D}$ and $3 \mathrm{D}$ cases as anticipated in the original work [22]. In particular, the difference between these two cases is about $20 \%$ for average velocity $\left\langle v_{a}\right\rangle$, and about $14 \%$ for average energy $\left\langle E_{a}\right\rangle$. However, the spectra in the nonrelativistic regime $v_{a} \ll c$ are dramatically different, see Fig. 2.

Lastly, it is instructive to compare our approximate analytical solution (A3) to the exact numerical solution. To do this, we plot the corresponding solutions in Fig. 3 with $R_{0}$ chosen to be $0.01 m_{a}^{-1}$ for demonstration purpose. We find the exact numerical solution in general has a steeper growth, and approaches the outer vacuum expectation value faster comparing to approximate solutions with $0 \leq \delta \lesssim 1$. Beyond $\delta \lesssim 1$ we find a best-fit solution at about $\delta=8$, but we should also note such solution badly violates the continuity and first-order differentiability at the transition zone $r \sim R_{\text {trans. }}$. We also plot $\delta=8$ in the flux spectrum Figs. 1, which again gives qualitatively consistent answer. 


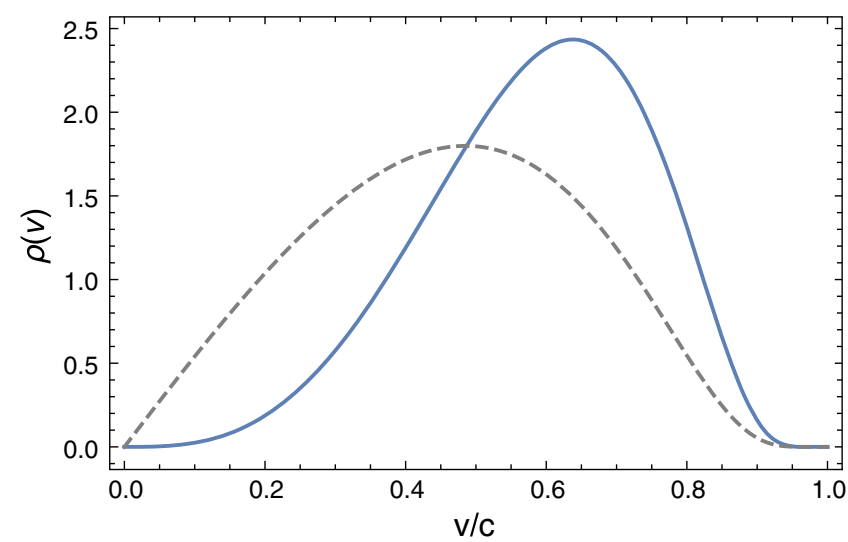

FIG. 2. Axion flux spectrum: $1 \mathrm{D}$ versus $3 \mathrm{D}$ case. Here $1 \mathrm{D}$ case (gray dotted) computed in [22] is compared with the 3D case (blue solid, $\delta=0$ ).

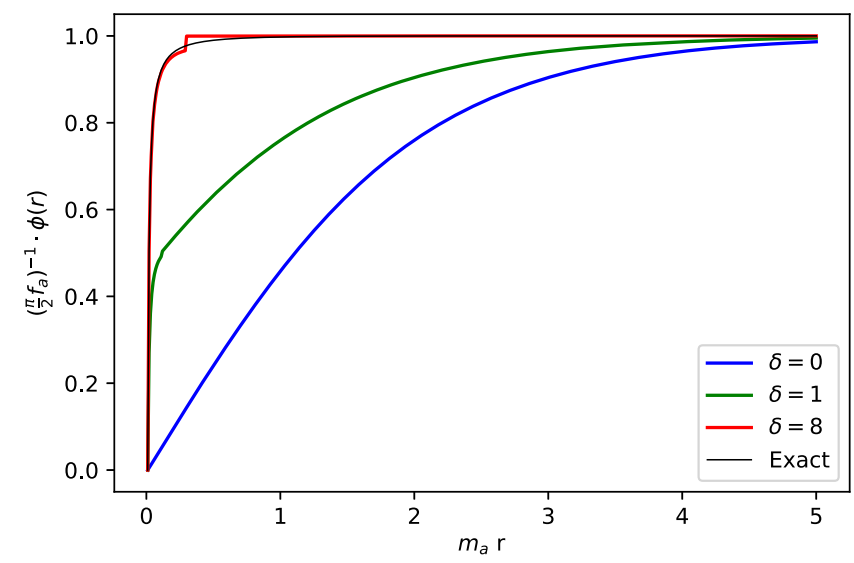

FIG. 3. Approximate and exact numerical solutions to Eq. (A2). Here we choose $m_{a} R_{0}=0.01$.
As a final remark, one should not consider the spectrum with $\delta=8$ any better than other values of $\delta$ for two reasons. First, solution with $\delta=8$ is not physical for its bad violation of continuity and smoothness. Second, even an exact numerical solution gives no better quantitative prediction because the effective Lagrangian (A1) is only a phenomenological model for qualitative analysis, see footnote 6 .

\section{APPENDIX B: ABOUT $K_{p, q}^{(l)}$}

This Appendix is devoted to proving $K_{p, q}^{(l)} \delta(p-q)=$ $\delta(p-q)$. Before we proceed to the proof, it is convenient to define two operators

$$
d_{l}=\frac{d}{d r}+\frac{l+1}{r}, \quad d_{l}^{\dagger}=-\frac{d}{d r}+\frac{l+1}{r} .
$$

And we will need some useful identities:

$$
\begin{gathered}
d_{l} d_{l}^{\dagger}\left[r j_{l}(r)\right]=r j_{l}(r) . \\
d_{l}^{\dagger}\left[r j_{l}(r)\right]=r j_{l+1}(r) \\
d_{l}^{\dagger} d_{l}=d_{l+1} d_{l+1}^{\dagger} \\
\int_{0}^{\infty}\left[d_{l} A(r)\right] \cdot B(r)=\int_{0}^{\infty} A(r) \cdot\left[d_{l}^{\dagger} B(r)\right] \\
(\text { if } A, B=0 \quad \text { at } r=0, \infty)
\end{gathered}
$$

Now we are able to prove by induction. First, the proof in case of $l=0$ is quite trivial:

$$
\begin{aligned}
K_{p, q}^{(0)} & =\lim _{L \rightarrow \infty} \frac{\int_{0}^{L} d r \sin (p r) \sin (q r) \frac{1}{2}\left[3\left(\frac{1}{v} \phi_{w, R_{0}}(r)\right)^{2}-1\right]}{\int_{0}^{L} d r \sin (p r) \sin (q r)} \\
& =\lim _{L \rightarrow \infty} \frac{\int_{0}^{L} d r[\cos (p-q) r-\cos (p+q) r] \frac{1}{2}\left[3\left(\frac{1}{v} \phi_{w, R_{0}}(r)\right)^{2}-1\right]}{\int_{0}^{L} d r[\cos (p-q) r-\cos (p+q) r]} .
\end{aligned}
$$

Up to this point, we note that $K_{p, q}^{(0)}$ is always finite for any positive $p, q>0$. Now, if we multiply both sides by $\delta(p-q)$

$$
K_{p, q}^{(0)} \delta(p-q)=\delta(p-q) \lim _{L \rightarrow \infty} \frac{\int_{0}^{L} d r[1-\cos (p+q) r] \frac{1}{2}\left[3\left(\frac{1}{v} \phi_{w, R_{0}}(r)\right)^{2}-1\right]}{\int_{0}^{L} d r[1-\cos (p+q) r]}=\delta(p-q)
$$

where we know the integral is quickly dominant by the term $\int_{0}^{L} d r \cdot 1$, so that the fraction in the limit $L \rightarrow \infty$ gives trivial result. 
Now, we want to show that $K_{p, q}^{(l)} \delta(p-q)=K_{p, q}^{(l+1)} \delta(p-q)$ for all $l=0,1,2 \ldots$ First, let us see that

$$
\begin{aligned}
K_{p, q}^{(l)}= & \lim _{L \rightarrow \infty} \frac{\int_{0}^{L} d r \cdot d_{l} d_{l}^{\dagger}\left[p r j_{l}(p r)\right] \cdot d_{l} d_{l}^{\dagger}\left[q r j_{l}(q r)\right] \cdot \frac{1}{2}\left[3\left(\frac{1}{v} \phi_{w, R_{0}}(r)\right)^{2}-1\right]}{\int_{0}^{L} d r \cdot d_{l} d_{l}^{\dagger}\left[p r j_{l}(p r)\right] \cdot d_{l} d_{l}^{\dagger}\left[q r j_{l}(q r)\right]} \\
= & \lim _{L \rightarrow \infty} \frac{\int_{0}^{L} d r \cdot p r j_{l+1}(p r) \cdot d_{l}^{\dagger} d_{l}\left[q r j_{l+1}(q r)\right] \cdot \frac{1}{2}\left[3\left(\frac{1}{v} \phi_{w, R_{0}}(r)\right)^{2}-1\right]}{\int_{0}^{L} d r \cdot p r j_{l+1}(p r) \cdot d_{l}^{\dagger} d_{l}\left[q r j_{l+1}(q r)\right.} \\
& -\lim _{L \rightarrow \infty} \frac{\int_{0}^{L} d r \cdot p r j_{l+1}(p r) \cdot q r j_{l+1}(q r) \cdot \frac{1}{2} \frac{d}{d r}\left[3\left(\frac{1}{v} \phi_{w, R_{0}}(r)\right)^{2}-1\right]}{\int_{0}^{L} d r \cdot p r j_{l+1}(p r) \cdot d_{l}^{\dagger} d_{l}\left[q r j_{l+1}(q r)\right]} \\
= & K_{p, q}^{(l+1)}-\lim _{L \rightarrow \infty} \frac{\int_{0}^{L} d r \cdot r^{2} j_{l+1}(p r) \cdot j_{l+1}(q r) \cdot \frac{1}{2} \frac{d}{d r}\left[3\left(\frac{1}{v} \phi_{w, R_{0}}(r)\right)^{2}-1\right]}{\int_{0}^{L} d r \cdot r^{2} j_{l+1}(p r) \cdot j_{l+1}(q r)},
\end{aligned}
$$

where we have applied Eqs. (B2a) to (B2d) in the intermediate steps. Again, the integral is finite for any $p, q>0$. Note that in the last line if we set $p=q$, the second term must vanish. It is because the numerator is obviously finite, while the denominator tends to infinity in the large $L$ limit as indicated by Eq. (A12a). Thus, we conclude

$$
K_{p, q}^{(l)} \delta(p-q)=\delta(p-q)
$$

for all $l=0,1,2,3 \ldots$ as expected.

[1] R. D. Peccei and H. R. Quinn, Phys. Rev. D 16, 1791 (1977); S. Weinberg, Phys. Rev. Lett. 40, 223 (1978); F. Wilczek, Phys. Rev. Lett. 40, 279 (1978).

[2] J. E. Kim, Phys. Rev. Lett. 43, 103 (1979); M. A. Shifman, A. I. Vainshtein, and V. I. Zakharov, Nucl. Phys. B166, 493 (1980).

[3] M. Dine, W. Fischler, and M. Srednicki, Phys. Lett. 104B, 199 (1981); A. R. Zhitnitsky, Yad. Fiz. 31, 497 (1980) [Sov. J. Nucl. Phys. 31, 260 (1980)].

[4] K. van Bibber and L. J. Rosenberg, Phys. Today 59, No. 8, 30 (2006).

[5] S. J. Asztalos, L. J. Rosenberg, K. van Bibber, P. Sikivie, and K. Zioutas, Annu. Rev. Nucl. Part. Sci. 56, 293 (2006).

[6] Pierre Sikivie, Lect. Notes Phys. 741, 19 (2008).

[7] G. G. Raffelt, Lect. Notes Phys. 741, 51 (2008).

[8] P. Sikivie, Int. J. Mod. Phys. A 25, 554 (2010).

[9] L. J. Rosenberg, Proc. Natl. Acad. Sci. U.S.A. 112, 12278 (2015).

[10] P. W. Graham, I. G. Irastorza, S. K. Lamoreaux, A. Lindner, and K. A. van Bibber, Annu. Rev. Nucl. Part. Sci. 65, 485 (2015).

[11] D. J. E. Marsh, Phys. Rep. 643, 1 (2016).

[12] A. Ringwald, Proc. Sci. NOW2016 (2016) 081 [arXiv: 1612.08933].

[13] J. Preskill, M. B. Wise, and F. Wilczek, Phys. Lett. 120B, 127 (1983); L. Abbott and P. Sikivie, Phys. Lett. 120B, 133 (1983); M. Dine and W. Fischler, Phys. Lett. 120B, 137 (1983).

[14] S. Chang, C. Hagmann, and P. Sikivie, Phys. Rev. D 59, 023505 (1998).
[15] T. Hiramatsu, M. Kawasaki, K. Saikawa, and T. Sekiguchi, Phys. Rev. D 85, 105020 (2012); 86, 089902(E) (2012).

[16] M. Kawasaki, K. Saikawa, and T. Sekiguchi, Phys. Rev. D 91, 065014 (2015).

[17] L. Fleury and G. D. Moore, J. Cosmol. Astropart. Phys. 01 (2016) 004.

[18] M. Gorghetto, E. Hardy, and G. Villadoro, J. High Energy Phys. 07 (2018) 151.

[19] V. B. Klaer and G. D. Moore, J. Cosmol. Astropart. Phys. 11 (2017) 049.

[20] P. Sikivie, Phys. Rev. Lett. 51, 1415 (1983); 52, 695(E) (1984).

[21] S. Andriamonje et al. (CAST Collaboration), J. Cosmol. Astropart. Phys. 04 (2007) 010.

[22] H. Fischer, X. Liang, Y. Semertzidis, A. Zhitnitsky, and K. Zioutas, Phys. Rev. D 98, 043013 (2018).

[23] L. DiLella and K. Zioutas, Astropart. Phys. 19, 145 (2003).

[24] A. R. Zhitnitsky, J. Cosmol. Astropart. Phys. 10 (2003) 010.

[25] X. Liang and A. Zhitnitsky, Phys. Rev. D 94, 083502 (2016).

[26] S. Ge, X. Liang, and A. Zhitnitsky, Phys. Rev. D 96, 063514 (2017).

[27] S. Ge, X. Liang, and A. Zhitnitsky, Phys. Rev. D 97, 043008 (2018).

[28] A. Zhitnitsky, J. Cosmol. Astropart. Phys. 10 (2017) 050.

[29] N. Raza, L. van Waerbeke, and A. Zhitnitsky, Phys. Rev. D 98, 103527 (2018).

[30] S. L. Adler, Phys. Rev. D 79, 023505 (2009).

[31] X. Xu and E. R. Siegel, arXiv:0806.3767. 
[32] I. B. Khriplovich and D. L. Shepelyansky, Int. J. Mod. Phys. D 18, 1903 (2009); I. B. Khriplovich, Int. J. Mod. Phys. D 20, 17 (2011).

[33] J. Lundberg and J. Edsjo, Phys. Rev. D 69, 123505 (2004).

[34] A. H. G. Peter, Phys. Rev. D 79, 103531 (2009); 79, 103533 (2009); J. Edsjo and A. H. G. Peter, arXiv: 1004.5258 .

[35] G. Raffelt and L. Stodolsky, Phys. Rev. D 37, 1237 (1988).

[36] A. N. Ioannisian, N. Kazarian, A. J. Millar, and G. G. Raffelt, J. Cosmol. Astropart. Phys. 09 (2017) 005.

[37] S. Bertolucci, K. Zioutas, S. Hofmann, and M. Maroudas, Phys. Dark Universe 17, 13 (2017).
[38] A. Zhitnitsky, Phys. Dark Universe 22, 1 (2018).

[39] V. V. Flambaum and A. R. Zhitnitsky, Phys. Rev. D 99, 023517 (2019).

[40] M. M. Forbes and A. R. Zhitnitsky, J. High Energy Phys. 10 (2001) 013.

[41] M. Abramowitz, in Handbook of Mathematical Functions, edited by M. Abramowitz and I. A. Stegun, NBS, Applied Mathematics Series Vol. 55 (U.S. Government Printing Office, Washington, DC, 1972), Chap. 15.

[42] M. Abramowitz, in Handbook of Mathematical Functions, edited by M. Abramowitz and I. A. Stegun, NBS, Applied Mathematics Series-55 (1972), Chap. 6.

[43] N. Michel and M. V. Stoitsov, Comput. Phys. Commun. 178, 535 (2008). 